\title{
1 Synaptic accumulation of FUS triggers age-dependent 2 misregulation of inhibitory synapses in ALS-FUS mice
}

3

4 Sonu Sahadevan $^{1^{*}}$, Katharina M. Hembach ${ }^{1,2^{*}}$, Elena Tantardini ${ }^{1}$, Manuela Pérez-Berlanga ${ }^{1}$, 5 Marian Hruska-Plochan ${ }^{1}$, Julien Weber ${ }^{1}$, Petra Schwarz ${ }^{3}$, Luc Dupuis ${ }^{4}$, Mark D. Robinson ${ }^{2}$, 6 Pierre De Rossi ${ }^{1}$, Magdalini Polymenidou ${ }^{1, \#}$

7

8

\section{Abstract}

${ }^{1}$ Department of Quantitative Biomedicine, University of Zurich

${ }^{2}$ Department of Molecular Life Sciences and SIB Swiss Institute of Bioinformatics, University of Zurich

${ }^{3}$ Institute of Neuropathology, University Hospital Zurich

${ }^{4}$ Inserm, University of Strasbourg

*These authors contributed equally to this work

\#Author for correspondence: magdalini.polymenidou@uzh.ch 
45 FUS is a primarily nuclear RNA-binding protein with important roles in RNA processing and 46 transport. FUS mutations disrupting its nuclear localization characterize a subset of 47 amyotrophic lateral sclerosis (ALS-FUS) patients, through an unidentified pathological 48 mechanism. FUS regulates nuclear RNAs, but its role at the synapse is poorly understood. 49 Here, we used super-resolution imaging to determine the physiological localization of 50 extranuclear, neuronal FUS and found it predominantly near the vesicle reserve pool of 51 presynaptic sites. Using CLIP-seq on synaptoneurosome preparations, we identified 52 synaptic RNA targets of FUS that are associated with synapse organization and plasticity. 53 Synaptic FUS was significantly increased in a knock-in mouse model of ALS-FUS, at 54 presymptomatic stages, accompanied by alterations in density and size of GABAergic synapses. RNA-seq of synaptoneurosomes highlighted age-dependent dysregulation of glutamatergic and GABAergic synapses. Our study indicates that FUS accumulation at the synapse in early stages of ALS-FUS results in synaptic impairment, potentially representing an initial trigger of neurodegeneration.

Keywords: FUS, ALS-FUS, neurodegeneration, RNA-binding proteins, synaptic function, 62 RNA transport, local translation, CLIP-seq, synaptoneurosomes, super-resolution 63 microscopy 
84 FUS (Fused in sarcoma) is a nucleic acid binding protein involved in several processes of 85 RNA metabolism ${ }^{1}$. Physiologically, FUS is predominantly localized to the nucleus ${ }^{2}$ via active transport by transportin (TNPO) ${ }^{3}$ and it can shuttle to the cytoplasm by passive diffusion ${ }^{4,5}$.

87 In amyotrophic lateral sclerosis (ALS) and frontotemporal dementia (FTD), FUS mislocalizes 88 to the cytoplasm where it forms insoluble aggregates ${ }^{6-8}$. In ALS, cytoplasmic mislocalization 89 of FUS is associated with mutations that are mainly clustered in the proline-tyrosine nuclear 90 localization signal (PY-NLS) at the C-terminal site of the protein ${ }^{9}$ and lead to mislocalization 91 of the protein to the cytosol. However, in FTD, FUS mislocalization occurs in the absence of 92 mutations $^{10}$. FUS is incorporated in cytoplasmic stress granules ${ }^{5,11}$ and undergoes 93 concentration-dependent, liquid-liquid phase separation ${ }^{12,13}$, which is modulated by binding 94 of TNPO and arginine methylation of FUS ${ }^{14-17}$. This likely contributes to the role of FUS in 95 forming specific identities of ribonucleoprotein (RNP) granules ${ }^{18,19}$ and in transporting RNA cargos $^{20}$, which is essential for local translation in neurons ${ }^{21}$.

97 Despite the central role of FUS in neurodegenerative diseases, little is known about its function in specialized neuronal compartments, such as synapses. FUS was shown to mediate RNA transport ${ }^{20}$ and is involved in stabilization of RNAs that encode proteins with important synaptic functions ${ }^{22}$, such as GluA1 and SynGAP1 ${ }^{23,24}$. While the presence of FUS protein in synaptic compartments has been confirmed, its exact subsynaptic localization is debated. Diverging results described the presence of FUS at the pre-synapses in close proximity to synaptic vesicles ${ }^{25-27}$, but also in dendritic spines ${ }^{20}$ and in association with the postsynaptic density ${ }^{28}$. Confirming a functional role of FUS at the synaptic sites, behavioral and synaptic morphological changes have been observed upon depletion of FUS in mouse models $^{23,29,30}$. Notably, mouse models associated with mislocalization of FUS exhibited reduced axonal translation contributing to synaptic impairments ${ }^{31}$. Synaptic dysfunction has been suggested to be the early event of several neurodegenerative disorders including ALS and $\mathrm{FTD}^{32-36}$. The disruption of RNA-binding proteins (RBPs) and RNA regulation could be a central cause of synaptic defects in these disorders.

111 Previous studies identified nuclear RNA targets of FUS with different cross-linking 112 immunoprecipitation and high-throughput sequencing (CLIP-seq) approaches ${ }^{22,37-41}$.

113 Collectively, these findings showed that FUS binds mainly introns, without a strong 114 sequence specificity, but a preference for either GU-rich regions ${ }^{22,38,40,41}$, which is mediated 115 via its zinc finger $(\mathrm{ZnF})$ domain, or a stem-loop $\mathrm{RNA}^{37}$ via its RNA recognition motif ${ }^{42}$. FUS 116 often binds close to alternatively spliced exons, highlighting its role in splicing 117 regulation ${ }^{22,38,39}$. CLIP-seq studies also identified RNAs bound by FUS at their 3' 
118 untranslated regions (3'UTRs) and exons ${ }^{22,39,41}$, suggesting a direct role of FUS in RNA

119 transport and regulating synaptic mRNA stability ${ }^{23,24}$ and polyadenylation ${ }^{40}$. However, a

120 precise list of synaptic RNAs directly regulated by FUS is yet to be identified.

121 In this study, we focused on understanding the role of synaptic FUS in RNA homeostasis

122 and the consequences of ALS-causing mutations in FUS on synaptic maintenance. Using

123 super-resolution imaging, we confirmed the presence of FUS at the synapse. FUS was

124 found at both excitatory and inhibitory synapses, was enriched at the presynapse and rarely

125 associated with postsynaptic structures. Synaptoneurosome preparations from adult mouse

126 cortex, coupled with CLIP-seq uncovered specific synaptic RNA targets of FUS.

127 Computational analyses revealed that most of these targets were associated with both

128 glutamatergic and GABAergic networks. In a heterozygous knock-in FUS mouse model,

129 which harbors a deletion in the NLS of FUS allele, thereby mimicking the majority of ALS-

130 causing mutations ${ }^{43}$, we found significant increase of synaptic FUS localization. To test the

131 effect of this elevation in synaptic FUS, we investigated the synaptic organization of the

132 hippocampus, which is enriched in glutamatergic and GABAergic synapses, and found mild

133 and transient changes. However, RNA-seq analysis revealed age-dependent alterations of

134 synaptic RNA composition including glutamatergic and GABAergic synapses. Our data

135 indicate that early synaptic alterations in the GABAergic network precede motor impairments

136 in these ALS-FUS mice ${ }^{43}$, and may trigger early behavioral dysfunctions, such as

137 hyperactivity and social disinhibition that these mice develop (Scekic-Zahirovic, Sanjuan-

138 Ruiz et al., co-submitted manuscript).

139 Altogether, our results demonstrate a critical role for FUS in synaptic RNA homeostasis via

140 direct association with specific synaptic RNAs, such as Gabra1, Grin1 and others. Our study

141 indicates that enhanced synaptic localization of FUS in early stages of ALS-FUS results in

142 synaptic impairment, potentially representing the initial trigger of neurodegeneration.

143 Importantly, we show that increased localization of FUS at the synapses, in the absence of

144 aggregation, suffices to cause synaptic impairment.

145

\section{Results}

147 FUS is enriched at the presynaptic compartment of mature cortical and hippocampal 148 neurons

149 While FUS has been shown at synaptic sites, its exact subsynaptic localization is debated.

150 Some studies described a presynaptic enrichment of FUS in cortical neurons and 151 motoneurons ${ }^{25,27}$, whereas others have shown an association of FUS with postsynaptic 152 density (PSD) $\operatorname{sites}^{20,28}$. To clarify the precise localization of FUS at the synapses, we first 153 performed confocal analysis in mouse cortex (Fig. 1a-b) and hippocampus (Supplementary 
154 Fig. 1a-b), which confirmed the presence of extranuclear FUS clusters along dendrites and 155 axons (identified with MAP2 and PNF, respectively) and associated with synaptic markers 156 (Synapsin1 and PSD95). To determine the precise subsynaptic localization of FUS, we used 157 super-resolution microscopy (SRM) imaging of mouse hippocampal and cortical synapses. 158 We first explored the distribution of FUS between excitatory and inhibitory synapses of cortical and hippocampal neurons (Fig. 1c). STED (Stimulated emission depletion) microscopy was used to precisely determine the localization of FUS clusters compared to synaptic markers: VGAT was used as a marker for inhibitory synapses and PSD95 for excitatory synapses. Image analysis was used to calculate the distance of the closest neighbor (Supplementary Fig. 1c). Only FUS clusters within $200 \mathrm{~nm}$ from a synaptic marker were considered for this analysis. Our results showed that extranuclear FUS preferentially associates with excitatory synapses, with $46 \%$ of the detected ones containing FUS, while only $20 \%$ of analyzed inhibitory synapses showing FUS positivity (t-test, $\mathrm{p}=0.0016)$ (Fig. 1d).

To better define the precise localization of FUS within the synapse, cortical and hippocampal primary cultures were immunolabeled for FUS along with pre- and postsynaptic markers (Fig. 1e and Supplementary Fig. 1d-e) and their relative distance was analyzed. At the presynapse, Synapsin 1 was used to label the vesicle reserve pool ${ }^{44}$, and Bassoon to label the presynaptic active zone ${ }^{45}$. At the postsynaptic site, GluN2B, subunit of NMDA receptors, and GluA1, subunit of AMPA receptors, were used to label glutamatergic synapses. PSD95 was used to label the postsynaptic density zone ${ }^{46}$. Distribution of FUS at the synapse showed a closer association with Synapsin 1 compared to Bassoon, GluA1, BiP (ER marker) and GluN2B (Supplementary Fig. 1f-g). FUS also appeared to be closer to Bassoon compared to PSD95 (Supplementary Fig. 1f-g). A subset of FUS was also localized at the spine (Fig. 1e). To strengthen our analyses and to refine the precise localization of FUS, the relative proportion of FUS within $100 \mathrm{~nm}$ was compared for each marker. Our results showed a preferential FUS localization at the presynaptic site (Fig. 1f) (t-test, $p=0.0006$ ), in accordance with previously reported data ${ }^{25,27}$. Within the presynaptic site (Fig. 19), FUS was significantly enriched in the Synapsin-positive area (One-way ANOVA, $p<0.0001$, posthoc Tukey, Syn1 vs. PSD95, $p<0.0001$; Syn1 vs. GluN2B, $p=0.0157$; Syn1 vs. GluA1, $p=0.454$; Syn1 vs. Bassoon, $p=0.0005)$. However, no significant difference was found with the $E R$ marker, suggesting that FUS could be localized between Synapsin 1 and ER at the FUS within $150 \mathrm{~nm}$ from the active presynaptic zone ${ }^{27}$, but highlight the presence of FUS also at the postsynaptic site, potentially explaining the apparently contradictory results of previous studies ${ }^{20,28}$. 


\section{Identification of synaptic RNA targets of FUS}

192 The role of FUS in the nucleus has been well studied and previously published CLIP-seq

193 data identified FUS binding preferentially on pre-mRNA, suggesting that these binding 194 events occur in the nucleus ${ }^{22,47-50}$. Given the confirmed synaptic localization of FUS (Fig. 1), 195 we wondered if a specific subset of synaptic RNAs are directly bound and regulated by FUS 196 in these compartments. Since synapses contain few copies of different RNAs and only a small fraction of the total cellular FUS is synaptically localized, RNAs specifically bound by FUS at the synapses are likely missed in CLIP-seq datasets from total brain. Therefore, we biochemically isolated synaptoneurosomes that are enriched synaptic fractions from mouse cortex to identify synapse-specific RNA targets of FUS. Electron microscopy analysis confirmed the morphological integrity of our synaptoneurosome preparations, which contained intact pre- and postsynaptic structures (Fig. 2a). Immunoblot showed an enrichment of synaptic markers (PSD-95, p-CAMKII, GluN2B, GluA1, SNAP25, NXRN1), absence of nuclear proteins (Lamin B1, Histone $\mathrm{H3}$ ) and presence of FUS in the synaptoneurosomes (Fig. 2b and Supplementary 2a). In addition, quantitative reverse transcription polymerase chain reaction (qRT-PCR) analysis showed enrichment of selected synaptic mRNAs (Fig. 2c).

208

Following a previously published method ${ }^{22,51}$, we used ultraviolet (UV) crosslinking on isolated synaptoneurosomes and total cortex from 1-month-old wild type mice to stabilize FUS-RNA interactions and to allow stringent immunoprecipitation of the complexes (Supplementary Fig. 2b). As FUS is enriched in the nucleus and only a small fraction of the protein is localized at the synapses, we prepared synaptoneurosomes from cortices of 200 mice to achieve sufficient RNA levels for CLIP-seq library preparation. The autoradiograph showed an RNA smear at the expected molecular weight of a single FUS molecule (70 kDa) and lower mobility complexes (above $115 \mathrm{kDa}$ ) that may correspond to RNAs bound by more than one FUS molecule or a heterogeneous protein complex (Fig. 2d). No complexes were immunoprecipitated in the absence of UV cross-linking or when using nonspecific IgGcoated beads. The efficiency of immunoprecipitation was confirmed by depletion of FUS in post-IP samples (Supplementary Fig. 2c). Finally, RNAs purified from the FUS-RNA complexes of cortical synaptoneurosomes and total cortex were sequenced and analyzed. We obtained 29,057,026 and 27,734,233 reads for the total cortex and cortical synaptoneurosome samples, respectively. $91 \%$ of the total cortex and $66 \%$ of the synaptoneurosome reads could be mapped to a unique location in the mouse reference genome (GRCm38) (Supplementary Fig. 2d). After removing PCR duplicates, we identified peaks using a previously published tool called CLIPper ${ }^{52}$, resulting in 619,728 total cortex and 408,918 synaptoneurosome peaks.

227 Before comparing the peaks in the two samples, we normalized the data to correct for 
228 different sequencing depths and signal-to-noise ratios ${ }^{53}$ (see Methods). This is especially

229 important in our case, because the synaptoneurosome sample should contain only a subset

230 of the FUS targets from total cortex. We wanted to filter the predicted peaks of the

231 synaptoneurosome sample to identify genomic regions with high log2 fold-change between

232 the synaptoneurosome and total cortex samples. Peaks with low number of reads (or no

233 reads) in the total cortex, but high read coverage in the synaptoneurosomes correspond to

234 regions that are putatively bound by FUS in the synapse. However, the observable number

235 of reads per RNA in each sample strongly depends on gene expression and the number of

236 localized RNA copies. Therefore, we did not want to use a simple read count threshold to

237 filter and identify synapse specific peaks. Instead, we fit a count model and computed peak-

238 specific $p$-values to test for differences between the synaptoneurosome and total cortex

239 CLIP-seq enrichment (Fig. 2e). The normalization highlights the expected association

240 between p-values (yellow) and log2 CPM (Fig. 2e).

241 We ranked the peaks by p-values and used a stringent cutoff of $1 e-5$ (Fig. 2e) to ensure

242 enrichment of synaptic FUS targets. Indeed, the resulting peaks were largely devoid of

243 intronic regions, but were enriched in exons and 3'UTRs, as was expected for synaptic FUS

244 targets, which are mature and fully processed RNAs (Fig. 2e and Supplementary Fig. 2g).

245 The same normalization and filtering of CLIPper peaks identified in the total cortex

246 highlighted RNAs primarily bound by FUS in the nucleus, where the vast majority of FUS

247 protein resides (Supplementary Fig. 2e). After selecting an equal number of top peaks as

248 obtained for the synaptoneurosome sample (1560 peaks in 517 genes), corresponding to a

249 p-value cutoff of 0.0029 (Supplementary Fig. 2f), we confirmed the previously reported ${ }^{22}$

250 preferential binding of FUS within intronic regions of pre-mRNAs (Fig. $\mathbf{2 g}$ and

251 Supplementary Fig. 2h).

252 The final list of synapse-specific FUS binding sites consists of 1560 peaks in 307 RNAs

253 (Supplementary Table 1), primarily localized to exons and 3'UTRs of RNAs specific to the

254 synapses. Among those, FUS peaks on the exon of Grin1 (Glutamate ionotropic NMDA type

255 subunit 1) and 3'UTR of a long isoform of Gabra1 (Gamma aminobutyric acid receptor

256 subunit alpha-1) were exclusively detected in synaptoneurosomes, but not in total cortex

257 (Fig. 2h-i). Direct binding of FUS to 3'UTR and exonic regions of its targets suggests a

258 potential role in regulating RNA transport, local translation and/or stabilization.

259

260 Synaptic FUS RNA targets encode essential protein components of synapse

261 We then wondered if the 307 synaptic FUS target RNAs were collectively highlighting any

262 known cellular localization and function. Most RNAs are localized to either the pre- or

263 postsynapse or they are known astrocytic markers (Fig. 2j). Among those are RNAs

264 encoding essential protein members of glutamatergic (Grin1, Gria2, Gria3) and GABAergic 
265

266

267

268

269

270

271

272

273

274

275

276

277

278

279

280

281

282

283

284

285

286

287

288

289

290

291

292

293

294

295

296

297

298

299

300

301

synapses (Gabra1, Gabrb3, Gabbr1, Gabbr2), transporters, as well as components of the calcium signaling pathway, which are important for plasticity of glutamatergic synapses. An overrepresentation analysis (ORA) comparing the synaptic FUS targets to all synaptic RNAs detected in cortical mouse synaptoneurosomes by RNA-seq (logCPM >1, 1-month-old mice), revealed that FUS targets were enriched for synaptic - both pre- and postsynaptic localization. Synaptic FUS target RNAs were enriched for gene ontology categories, such as transport, localization and trans-synaptic signaling, as well as signaling receptor binding and transmembrane transporter activity (Supplementary Fig. 2i).

Here we identified for the first time specific synaptic RNA targets directly bound by FUS, including those associated with glutamatergic and GABAergic networks. Our data suggests that FUS plays a critical role in maintaining synaptic integrity and organization.

\section{FUS binds GU-rich sequences at the synapse}

While FUS has been shown to be a relatively promiscuous RNA-binding protein, preference towards GU-rich motifs has been reported in previous CLIP-seq studies ${ }^{22,38,40,41}$, a binding mediated via its $\mathrm{ZnF}$ domain ${ }^{42}$. To understand if FUS binding to synaptic RNA targets follows the same modalities as its nuclear targets, we explored the sequence specificity of FUS in the synapse and predicted motifs with $\mathrm{HOMER}^{54}$, comparing the FUS peak sequences of cortical synaptoneurosomes and total cortex samples. In accordance with previous studies, we found a degenerate GU-rich motif for intronic FUS binding sites in the total cortex (Table 1). The sequences of the synaptic FUS peaks in exons and 5' UTRs revealed a "AGGUAAGU" motif which was only found in $11 \%$ and $6 \%$ of the peaks, respectively. We conclude that FUS does not have a stronger sequence preference in the synapse than in the nucleus.

\section{Increased synaptic localization of mutant FUS protein in Fus ${ }^{\Delta N L S /+}$ mice}

In order to explore synaptic impairments associated with FUS mislocalization, we used the Fus $^{\triangle N L S /+}$ mouse model ${ }^{55}$. This mouse model shows partial cytoplasmic mislocalization of FUS due to a lack of the nuclear localization (NLS) in one copy of the FUS allele, closely mimicking ALS-causing mutations reported in patients. Taking advantage of two antibodies that recognize either total FUS (both full length and mutant) or only the full length protein (Fig. 3a), we assessed FUS protein levels in synaptoneurosomes isolated from Fus ${ }^{\triangle N L S /+}$ mice and wild type $\left(\mathrm{Fus}^{+/+}\right)$of 1 and 6 months of age. We detected higher levels of total FUS in synaptoneurosomes from $F u s^{\Delta N L S /+}$ at both ages compared to Fus ${ }^{+/+}$(Fig. 3b-c, Supplementary Fig. 3a-b). However, full length FUS levels were decreased in synaptoneurosomes of $\mathrm{Fus}^{\Delta \mathrm{NLS/+}}$ compared to $\mathrm{Fus}^{+/+}$indicating that the truncated FUS protein is misaccumulated at the synaptic sites of $F u s^{\Delta N L S /+}$ mice. 
302 Confirming our biochemical evidence, immunofluorescence analyses of Fus ${ }^{\Delta N L S /+}$ mice

303 showed higher levels of FUS in dendritic compartments of CA1 pyramidal cells. Fus ${ }^{+/+}$mice

304 at both 1 month (Supplementary Fig. 3c-d) and 6 months of age (Fig. 3d-e) showed

305 prominent expression of FUS in the nucleus. High magnification images highlighted the 306 presence of FUS at the synapses, identified by co-labeling with Synapsin1. Fus ${ }^{\triangle N L S /+}$ mice at

3071 (Supplementary Fig. 3c-d) and 6 months of age (Fig. 3d-e) showed higher levels of FUS

308 within the dendritic tree (identified with MAP2) and at the synapse compared to Fus ${ }^{+/+}$mice,

309 confirming our previous quantifications by immunoblot.

310 Dysregulation of inhibitory synapses in Fus ${ }^{\Delta N L S /+}$ mouse model

311 To explore a possible synaptic disorganization associated with mislocalization of FUS, we 312 performed synaptic density and size analyses. Based on evidence that the 313 hippocampal/prefrontal cortex connectome participates in memory encoding and recalling ${ }^{56}$ 314 and that CA1 hippocampal excitatory and inhibitory synapses are highly similar to the 315 cortical synapses ${ }^{57-60}$, we explored the possible synaptic changes triggered by FUS 316 mislocalization in the CA1 hippocampal region. We analyzed both $F u s^{+/+}$and Fus ${ }^{\Delta N L S /+}$ mice, 317 using presynaptic and postsynaptic markers. Density and area analyses were performed as 318 shown in Supplementary Fig. 3e. At the presynapse, we quantified the density of the 319 SNARE associated protein SNAP25 ${ }^{61}$ (synaptic RNA target of FUS) and the presynaptic 320 active zone marker Bassoon ${ }^{45}$. The density of inhibitory synapses was assessed using 321 VGAT $^{62}$ (presynaptic). At the postsynapse, we quantified the density of postsynaptic 322 glutamatergic receptor GluN1 ${ }^{63}$ (synaptic RNA target of FUS and obligatory subunit of all 323 NMDAR) and GluA1 ${ }^{64}$ (obligatory subunit of AMPAR), as well as postsynaptic GABAergic 324 receptors containing a1 subunit $\left(G_{A B A} \alpha 1\right.$; synaptic RNA target of FUS) and a3

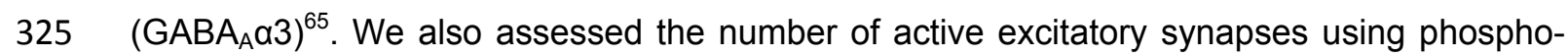
326 CaMKII (pCaMKII) as well as functional inhibitory synapses using Gephyrin ${ }^{66}$.

327 At 1 month of age in Fus ${ }^{\triangle N L S /+}$ mice, we did not observe significant changes at the 328 presynaptic site, suggesting a normal axonal and axon terminal development and functions. 329 However, at the postsynaptic sites, we observed a significant increase of NMDAR $330(p=0.0219)$ and a significant decrease of $G_{A B A_{A} \alpha 3}$ receptors $(p=0.0156)$ (Fig. 3f-g, 331 Supplementary Fig. 3f and Table 2). Moreover at 1 month of age, Fus ${ }^{\triangle N L S /+}$ mice showed 332 significantly more NMDAR located at the extrasynaptic site $(p=0.0433)$ (Fig. 3h). 333 Interestingly, the size of the $\mathrm{GABA}_{A} \alpha 3$ clusters was significantly decreased in Fus ${ }^{\triangle N L S /+}$ mice $334(p=0.0053)$ at 1 month of age (Fig. 3f, i, Supplementary Fig. 3h and Table 3). We did not 335 record changes in the number of Synapsin1, Bassoon, SNAP25, VGAT, GluA1, GABA $A_{A} 1$, 336 Gephyrin or pCaMKII, suggesting either an increase of silent synapses, immature synapses 
337 or an increase of the number of NMDAR in the dendritic shaft together with a decrease of

$338 \mathrm{GABA}_{A} \alpha 3$ synaptic clustering. These results suggested a hyperexcitability profile during 339 developmental stages.

340 At 6 months of age, we did not observe significant changes in the density of pre or 341 postsynaptic markers (Fig. 3f-g and Supplementary Fig. 3g), suggesting a normal 342 maturation of the synaptic network despite developmental synaptic dysregulation described 343 above. However, SNAP25 $(p=0.085)$ and VGAT $(p=0.0792)$ trended towards an increased 344 density, suggesting a potential alteration at inhibitory presynaptic sites (Supplementary Fig. $3453 \mathrm{~g}$ and Table 2). This interpretation was confirmed by an increase of the area of the 346 presynaptic marker VGAT $(p=0.0028)$ and of the size of $G_{A B A} \alpha 3$ clusters at the 347 postsynaptic site ( $p=0.0166$ ) (Fig. 3i, Supplementary Fig. 3i and Table 3), while GluN1 348 clusters appeared unaffected. Increase in VGAT suggested an elevated number of 349 presynaptic GABAergic vesicles, which was confirmed by EM analyses in older mice 350 (Scekic-Zahirovic, Sanjuan-Ruiz et al., co-submitted manuscript). Correlatively, increase of $351 \mathrm{GABA}_{A} \alpha 3$ cluster size suggested an increase in the trafficking of $G A B A_{A} R$ at the 352 postsynaptic site. This occurred, however, without an increase of the anchoring protein 353 Gephyrin, suggesting instable structure of the inhibitory postsynaptic sites. Altogether, our 354 results show alterations of both glutamatergic and GABAergic synapses during 355 developmental synaptogenesis (1 month of age), while only GABAergic synapses appeared 356 affected at a later time point (6 months of age). This suggests a potential role for FUS in 357 synaptogenesis and network wiring and synaptic maintenance, with a selective exacerbation 358 of inhibitory synaptic defects with age.

359

Fus $^{\Delta N L S /+}$ mice show age-dependent synaptic RNA alterations

361 FUS plays an essential role in RNA stabilization ${ }^{23,24}$ and transport ${ }^{20}$. Therefore, we used 362 RNA-seq to investigate the consequences of increased synaptic levels of mutated FUS in $363 \mathrm{Fus}^{\triangle N L S /+}$ mice (Fig. 4a). We isolated RNA from six biological replicates of 364 synaptoneurosomes and paired total cortex samples from $\mathrm{Fus}^{+/+}$and $F u s^{\Delta N L S /+}$ mice at 1 and 3656 months of age and prepared poly-A-selected libraries for high-throughput sequencing. As 366 a control, we also sequenced the nuclear fraction from 4 biological replicates of $\mathrm{Fus}^{+/+}$mice 367 at 1 month of age. For quality control, we computed principal components of all samples and 368 all expressed genes (see methods for details) and found a clustering by sample condition 369 and age (Supplementary Fig. 4b-c).

370 We compared the expressed genes in our synaptoneurosomes (15087 genes) with the 371 forebrain synaptic transcriptome ${ }^{67}$ (14073 genes) and the vast majority of detected RNAs 372 (13475) were identical between the two studies (Supplementary Fig. 4a). The small 
373 differences in the two transcriptomes can be explained by differences in the used

374 synaptoneurosome protocols and the brain region (frontal cortex versus forebrain).

375 We conducted four differential gene expression analyses, comparing Fus ${ }^{\Delta N L S /+}$ to $\mathrm{Fus}^{+/+}$

376 replicates separately for the total cortex and synaptoneurosomes at both time points (for full

377 lists see Supplementary Tables 2-5). A false discovery rate (FDR) cutoff of 0.05 was used

378 to define significant differential expression. Only three and five RNAs were differentially

379 expressed (DE) in the Fus ${ }^{\triangle N L S /+}$ samples of the total cortex at 1 and 6 months of age,

380 respectively (Supplementary Fig. $4 \mathbf{f}$ and Supplementary Tables 2-3). However, in the

381 synaptoneurosomes, we identified 11 and 594 RNAs differentially abundant at 1 and 6

382 months, respectively (Supplementary Tables 4-5). 136 RNAs were decreased and 485

383 RNAs were increased in the $F u s^{\Delta N L S /+}$ mice at 6 months of age compared to

384 synaptoneurosomes from $\mathrm{Fus}^{+/+}$mice (Fig. 4b). The significantly increased RNAs in

385 Fus $^{\triangle N L S /+}$ mice at 6 months were enriched in gene ontology (GO) categories such as

386 synaptic signaling, intrinsic component of membrane and transporter activity

387 (Supplementary Fig. 4d), while those that were decreased in abundance were associated

388 with cytoskeletal organization and RNA metabolism (Supplementary Fig. 4e).

389 At 6 months of age, the log2 fold changes of the altered RNAs are consistently negative or

390 positive in all Fus ${ }^{\Delta N L S /+}$ synaptoneurosome replicates (Fig. 4c). At 1 month of age, the log2

391 fold changes of the $F u s^{\triangle N L S /+}$ synaptoneurosome replicates are mostly neutral (white color on

392 the heatmap) indicating that alterations in RNA abundance are age-dependent and not

393 detectable as early as 1 month of age. In the total cortical samples at 6 months of age, some

394 of the replicates show a similar trend as the synaptoneurosome samples, but it seems that

395 the effects cannot be detected because synaptic RNAs are too diluted (Supplementary Fig.

396 4g). Overall, we found synapse-specific differential RNA abundance at 6 months in the

$397 F u s^{\triangle N L S /+}$ mice, but not in the total cortex.

398 While most of the 594 differentially abundant RNAs (Supplementary Table 5) were not 399 direct FUS targets, 33 altered RNAs are synaptic targets of FUS. The altered synaptic 400 transcriptome, along with the impaired expression of a subset of FUS RNA targets in $401 \mathrm{Fus}^{\Delta N L S /+}$ mice, suggests direct and indirect effects of mutant FUS at the synapses (Fig. 4d). 402 FUS targets with known synaptic functions that are altered in Fus ${ }^{\Delta N L S /+}$ are represented in 403 Fig. 4e. Most of those RNAs show exonic FUS binding on our CLIP-seq analysis 404 (Supplementary Fig. 5-6, Supplementary Table 1), with the exception of Gria 3, Spock1, 405 Spock2 (Supplementary Fig. 6b, f-g) and Gabra1 (Supplementary Fig. 7), which are 406 bound by FUS at their 3'UTR. Altered FUS targets include RNAs encoding presynaptic 407 vesicle associated proteins, transsynaptic proteins, membrane proteins, receptors 408 associated with glutamatergic and GABAergic pathways. Our results suggest that 409 mislocalization of FUS leads to mild alterations in the synaptic RNA profile that may affect 
410 synaptic signaling and plasticity. Our data indicate that synaptic RNA alterations may occur

411 at an asymptomatic age and represent one of the early events in disease pathogenesis.

412

\section{Discussion}

414 In this study, we identified for the first-time synaptic RNA targets of FUS combining cortical 415 synaptoneurosome preparations with CLIP-seq. Additionally, synaptic RNA levels were 416 found to be altered in a Fus ${ }^{\triangle N L S /+}$ mouse model at 6 months of age. Along with these results, 417 we assessed FUS localization at the synaptic site using a combination of super-resolution 418 microscopy approaches. Altogether, our results point to a critical role for FUS at the synapse 419 and indicate that increased synaptic FUS localization at presymptomatic stages of ALS-FUS 420 mice triggers early alterations of synaptic RNA content and misregulation of the GABAergic 421 network. These early synaptic changes mechanistically explain the behavioral dysfunctions 422 that these mice develop (Scekic-Zahirovic, Sanjuan-Ruiz et al., co-submitted manuscript).

423 RNA transport and local translation ensure fast responses with locally synthesized proteins 424 essential for plasticity ${ }^{21,68,69}$. CLIP-seq using synaptoneurosome preparations from mouse 425 cortex demonstrated that FUS not only binds nuclear RNAs, but also those that are localized 426 at the synapses. Both pre- and postsynaptic localization of the identified targets correlated 427 with the subcellular localization of FUS in both synaptic compartments. Moreover, by CLIP428 seq on synaptoneurosomes, we identified that FUS binds RNAs encoding GABA receptor 429 subunits (Gabra1, Gabrb3, Gabbr1, Gabbr2) and glutamatergic receptors (Gria2, Gria3, 430 Grin1) previously known to be localized at dendritic neuropils ${ }^{70}$. FUS binding on synaptic 431 RNAs is enriched on 3'UTRs and/or exonic regions, as revealed by our synaptoneurosome 432 CLIP-seq dataset, suggesting that FUS might play a role in regulating local translation or 433 transport of these targets.

434 Synaptic analyses at presymptomatic ages of $F u s^{\Delta N L S /+}$ mice revealed interesting changes. 435 Our results showed a major effect on inhibitory synapses at 1 and 6 months of age. We 436 explored $G A B A_{A} R$ density and found changes in a3-containing $G A B A_{A} R$. GABA $A_{A} \alpha 3$ is 437 expressed at the postsynaptic site of monoaminergic synapses ${ }^{71}$, and have been shown to 438 be involved in fear and anxiety behavior, and mutations in the Gabra3 subunit resulted in an 439 absence of inhibition behavior ${ }^{72-74}$. Changes in $G_{A B A} \alpha 3$ and not $G_{A B A} \alpha 1$-containing 440 receptor suggested that only monoaminergic neurons were affected in the Fus ${ }^{\triangle N L S /+}$ mouse 441 model. These results are well aligned with a contemporaneous study (Scekic-Zahirovic, 442 Sanjuan-Ruiz et al., co-submitted manuscript), which showed specific behavioral changes 443 that can be linked to monoaminergic networks. Interestingly at 1 month of age, Fus ${ }^{\triangle N L S /+}$ 444 mice showed an increase of NMDAR associated with a decrease in GABA $A_{A} \alpha 3$. These results 445 suggested a role for FUS during synaptogenesis in regulating postsynaptic receptor 
446 composition as previously suggested ${ }^{23,28,75}$. In 1-month-old Fus ${ }^{\Delta N L S /+}$ mice, NMDARs were

447 enriched at the extrasynaptic sites, which, together with the decrease in $G_{A B A} \alpha 3$,

448 suggested an hyperexcitability profile during development. We hypothesize that abnormal

449 activity during developmental stages could result in abnormal network connection. Fus ${ }^{\Delta N L S /+}$

450 mice at 6 months of age showed higher density of presynaptic inhibitory boutons, pointing

451 toward a compensatory mechanism at the GABAergic synapses to overcome the

452 hyperexcitability profile observed during development. Moreover at 6 months of age,

$453 \mathrm{Fus}^{\triangle N L S /+}$ mice also displayed higher density of SNAP25, present at both inhibitory and

454 excitatory synapses ${ }^{61,76}$, but we did not explore if this increase was specific for the

455 GABAergic network.

456 Interestingly, the cluster size of VGAT, which is involved in the transport of GABA in the 457 presynaptic vesicles ${ }^{77}$, was increased in $F u s^{\Delta N L S /+}$ mice at 6 months of age. Increase of the 458 cluster size would suggest that either more vesicles were present at the presynapse, or an 459 increase of VGAT protein per vesicle. We also observed an increase in $\mathrm{GABA}_{A} \alpha 3$ cluster 460 size and their density in 6-month-old Fus ${ }^{\Delta N L S /+}$ mice. Surprisingly, we did not observe an 461 increase in Gephyrin, a postsynaptic protein responsible for anchoring GABAR at the 462 postsynaptic site ${ }^{78,79}$. Gephyrin interacts at the postsynaptic site with GABAR at a ratio $1: 1^{80}$, 463 suggesting that inhibitory synapses in the $F u s^{\Delta N L S /+}$ model were unstable at 6 months of age 464 with an excess of GABAR poorly anchored at the postsynaptic site, which could lead to malfunction of the inhibitory network. In correlation, Fus ${ }^{\Delta N L S /+}$ mice showed behavioral changes overtime with disinhibition and hyperactivity behaviors as early as 4 months of age, associated with a decrease in the number of inhibitory neurons at 22-month-old (ScekicZahirovic, Sanjuan-Ruiz et al., co-submitted manuscript). Altogether, these results suggest that increased level of extranuclear FUS during development led to abnormal synaptogenesis affecting the GABAergic system over time.

471 Using the Fus ${ }^{\triangle N L S /+}$ mouse model, we found that accumulation of mislocalized mutant FUS at 472 the synapses altered the synaptic RNA content as early at 6 months of age. These 473 alterations include FUS target RNAs that are associated with glutamatergic (Grin1, Gria2, 474 Gria3) and GABAergic (Gabra1) synapses. These targets were found with increased 475 synaptic localization in $F u s^{\Delta N L S /+}$. An impairment of genes associated with the GABAergic 476 network in the frontal cortex of both young (5-month-old) and old (22-month-old) Fus ${ }^{\triangle N L S /+}$ 477 mice has been shown by an independent study (Scekic-Zahirovic, Sanjuan-Ruiz et al., co478 submitted manuscript). Importantly, this ALS-FUS mouse model developed behavioral 479 deficits, including hyperactivity and social disinhibition, suggesting defects in cortical 480 inhibition. Our data supports that phenotypic manifestations in $F u s^{\Delta N L S /+}$ mice could be due to 481 synaptic RNA alterations caused by mutant FUS at synapses. Moreover, mutant FUS482 associated synaptic RNA alterations precede in ALS-FUS mice as suggested in our data. 
483 However, the precise mechanism of how FUS regulates these targets is yet to be 484 determined.

485 CLIP-seq from synaptoneurosomes showed that FUS binds selectively to specific GABA 486 receptor subunits encoding mRNAs: Gabra1, Gabrb3, Gabbr1, Gabbr2. Other RNA-binding 487 proteins, such as fragile $X$ mental retardation protein (FMRP), Pumilio 1, 2 and cytoplasmic polyadenylation binding element binding protein (CPEB) have also been shown to bind 489 GABAR subunit mRNAs by CLIP-seq ${ }^{81}$. Whether all these proteins act in concert to locally regulate the expression of GABAR subunits at synapses needs to be investigated. Interestingly, FUS interacts with FMRP, a well-studied protein known to regulate local translation $^{82}$. Long 3' UTRs have been suggested to promote increased binding of RBPs and miRNAs which control the translation of these mRNAs ${ }^{83}$. Our CLIP-seq from synaptoneurosomes showed that FUS binds to the long 3' UTR containing isoform of Gabra1 (Supplementary Fig. 7) indicating that FUS may be directly involved in regulating the protein expression of Gabra1 at the synapses. Furthermore, we found increased levels of Gabra1 mRNA in synaptoneurosome preparations from Fus ${ }^{\Delta N L S /+}$ mice. It is important to study whether elevated levels of FUS at the synapse may directly impact Gabra1 levels via mRNA stabilization or local translation leading to altered regulation of inhibitory network. Overall, our findings highlight the role of FUS in synaptic RNA homeostasis possibly through regulating RNA transport, RNA stabilization and local translation.

511 Materials and Methods

\section{Experimental models}

514 Mice housing and breeding were in accordance with the Swiss Animal Welfare Law and in 515 compliance with the regulations of the Cantonal Veterinary Office, Zurich. We used 1- to 6516 month-old C57/BI6 mice or Fus ${ }^{+/} / F u s^{\Delta N L S /+}$ mice with genetic background (C57/BI6). Wild

517 type and heterozygous Fus ${ }^{\Delta N L S /+}$ mice with genetic background $(\mathrm{C} 57 / \mathrm{BI} 6)^{55}$ were bred and 518 housed in the animal facility of the University of Zurich. 


\section{Immunofluorescence staining for brain sections}

521 Mice were anesthetized by $\mathrm{CO}_{2}$ inhalation before perfusion with PBS containing $4 \%$

522 paraformaldehyde and $4 \%$ sucrose. Brains were harvested and post-fixed overnight in the

523 same fixative and then stored at $4^{\circ} \mathrm{C}$ in PBS containing $30 \%$ sucrose. Sixty $\mu \mathrm{m}$-thick coronal

524 sections were cut on a cryostat and processed for free-floating immunofluorescence

525 staining. Brain sections were incubated with the indicated primary antibodies for $48 \mathrm{~h}$ at $4^{\circ} \mathrm{C}$

526 followed by secondary antibodies for $24 \mathrm{~h}$ at $4^{\circ} \mathrm{C}$. The antibodies were diluted in $1 \mathrm{X}$ Tris

527 Buffer Saline solution containing 10\% donkey serum, 3\% BSA, and $0.25 \%$ Triton-X100.

528 Sections were then mounted on slides with Prolong Diamond (Life Technologies) before

529 confocal microscopy.

530

\section{STED super-resolution imaging and analysis}

532 Super-resolution STED (Stimulated emission depletion microscopy) images of FUS and 533 synaptic markers were acquired on a Leica SP8 3D, 3-color gated STED laser scanning 534 confocal microscope. Images were acquired in the retrospenial cortical area in the layer 5 535 and in the molecular layer of the hippocampal CA1 area. A $775 \mathrm{~nm}$ depletion laser was used 536 to deplete both 647 and 594 dyes. The powers used for depletion lasers, the excitation laser 537 parameters, and the gating parameters necessary to obtain STED resolution were assessed 538 for each marker. $1 \mu \mathrm{m}$-thick Z-stacks of $1024 \times 1024$-pixel images at $40 \mathrm{~nm}$ step size were 539 acquired at $1800 \mathrm{kHz}$ bidirectional scan rate with a line averaging of 32 and 3 frame 540 accumulation, using a 100X (1.45) objective with a digital zoom factor of 7.5 , yielding 15.15 541 nm pixels resolution.

542 STED microscopy data were quantified from at least 2 image stacks acquired from $2 \mathrm{Fus}^{+/+}$ 543 adult mice. The STED images were deconvolved using Huygens Professional software 544 (Scientific Volume Imaging). Images were subsequently analyzed using Imaris software.

545 Volumes for each marker were generated using smooth surfaces with details set up at 0.01

$546 \mathrm{~m}$. The diameter of the largest sphere was set up at $1 \mu \mathrm{m}$. Threshold background 547 subtraction methods were used to create the surface, and the threshold was calculated for 548 each marker and kept constant. Surfaces were then filtered by setting up the number of 549 voxels $>10$ and $<2000$ pixels. Closest neighbor distance was calculated using integrated 550 distance transformation tool in Imaris. Distances were then organized and statistically 551 analyzed using mean comparison and t-test comparison. Distances greater than $200 \mathrm{~nm}$ 552 were removed from the analysis, and average distance were analyzed.

553

\section{Neuronal primary cultures}


555 Primary neuronal cell cultures were prepared from postnatal (P0) pups. Briefly, hippocampus 556 and cortex were isolated. Hippocampi were treated with trypsin $(0.5 \% \mathrm{w} / \mathrm{v})$ in HBSS-Glucose

557 (D-Glucose, $0.65 \mathrm{mg} / \mathrm{ml}$ ) and triturated with glass pipettes to dissociate tissue in Neurobasal 558 medium (NB) supplemented with glutamine (2 mM), 2\% B27, 2.5\% Horse Serum, $100 \mathrm{U}$ 559 penicillin-streptomycin and D-Glucose $(0.65 \mathrm{mg} / \mathrm{ml})$. Hippocampal cells were then plated 560 onto poly-D-lysine coated $18 \times 18 \mathrm{~mm}$ coverslips (REF) at $6 \times 10^{4}$ cells $/ \mathrm{cm}^{2}$ for imaging, and 561 for biochemistry at high density $\left(8 \times 10^{4}\right.$ cells $\left./ \mathrm{cm}^{2}\right)$. Cells were subsequently cultured in 562 supplemented Neurobasal (NB) medium at $37^{\circ} \mathrm{C}$ under $5 \% \mathrm{CO}_{2}$, one-half of the medium 563 changed every 5 days, and used after 15 days in vitro (DIV). Cortex were dissociated and 564 plated similarly to hippocampal cells in NB supplemented with $2 \%$ B27, 5\% horse serum, $1 \%$ $565 \mathrm{~N} 2,1 \%$ glutamax, $100 \mathrm{U}$ penicillin-streptomycin and D-Glucose $(0.65 \mathrm{mg} / \mathrm{ml})$.

566

\section{Direct Stochastic Optical Reconstruction Microscopy (dSTORM)}

568 Super-resolution images were acquired on a Leica SR Ground State Depletion 3D / 3 color 569 TIRFM microscope with an Andor iXon Ultra 897 EMCCD camera (Andor Technology PLC). 570 DIV15-18 mouse primary neurons were fixed for $20 \mathrm{~min}$ in 4\% PFA - 4\% sucrose in PBS. 571 Primary antibodies were incubated overnight at $4 \%$ in PBS containing $10 \%$ donkey serum, $5723 \% \mathrm{BSA}$, and $0.25 \%$ Triton X-100. Secondary antibodies were incubated at RT for $3 \mathrm{~h}$ in the 573 same buffer. After 3 washes in PBS, the cells were re-fixed with $4 \%$ PFA for 5 min. The 574 coverslips were then washed over a period of 2 days at $4^{\circ} \mathrm{C}$ in PBS to remove non-specific 575 binding of the secondary antibodies. Coverslips were mounted temporarily in an oxygen 576 scavenger buffer (200mM phosphate buffer, $40 \%$ glucose, $1 \mathrm{M}$ cysteamine hydrochloride 577 (M6500 Sigma), 0.5mg/mL Glucose-oxydase, $40 \mathrm{ug} / \mathrm{mL}$ Catalase) to limit oxidation of the 578 fluorophores during image acquisition. The areas of capture were blindly selected by direct 579 observation in DIC. Images were acquired using a 160X (NA 1.43) objective in the TIRF 580 mode North direction with a penetration of $200 \mathrm{~nm}$. Far red channels (Alexa 647 or 660) 581 were acquired using a $642 \mathrm{~nm}$ laser. Red channels (Alexa 568 or 555) were acquired using 582 a $532 \mathrm{~nm}$ laser. Green channel (Alexa 488) was acquired using $488 \mathrm{~nm}$ laser. Images were 583 acquired in 2D. The irradiation intensity was adjusted until the single molecule detection 584 reached a frame correlation $<0.25$. Detection particle threshold was defined between 20-60 585 depending on the marker and adjusted to obtain a number of events per frame between 0 586 and 25. The exposure was maintained at $7.07 \mathrm{~ms}$ and the EM gain was set at 300 . The 587 power of depletion and acquisition was defined for each marker and kept constant during 588 acquisition. The number of particles collected were maintained constant per markers and 589 between experiments. At least 3 independent cultures or coverslips were imaged per 590 marker. 


\section{Super-resolution image processing and analysis}

593 Raw GSD images were processed using a custom-made macro in Fiji to remove

594 background by subtraction of a running median of frames (300 renewed every 300 frames)

595 and subtracting the previously processed image once background was removed ${ }^{84}$. A blur

596 (0.7-pixel radius) per slice prior to median subtraction was applied to reduce the noise

597 further. These images were then processed using Thunderstorm plugin in Imagej. Image

598 filtering was performed using Wavelet filter (B-spline, order 3/scale2.0). The molecules were

599 localized using centroid of connected components, and the peak intensity threshold was

600 determined per marker/dye to maintain an $X Y$ uncertainty $<50$. Sub-pixel localization of

601 molecules was performed using PSF elliptical gaussian and least squared fitting methods

602 with a fitting radius of 5 pixels and initial sigma of 1.6 pixels. Images were analyzed using

603 Bitplane Imaris software v.9.3.0 (Andor Technology PLC). Volumes for each marker were

604 generated using smooth surfaces with details set up at 0.005 . The diameter of the largest

605 sphere was set up at $1 \mu \mathrm{m}$. A threshold background subtraction method was used to create

606 the surface and threshold was calculated and applied to all the images of the same

607 experiment. Surfaces were then filtered by setting up the area between $0.01-1 \mu \mathrm{m}^{2}$. The

608 closest neighbor distance was processed using the integrated distance transformation tool in

609 Imaris. Distances were then organized and statistically analyzed using median comparison

610 and ANOVA and Fisher's Least Significant Difference (LSD) test. Distances greater than 100

$611 \mathrm{~nm}$ were removed from the analysis, and average distance were analyzed.

612

\section{Preparation of synaptoneurosomes from mouse brain tissues}

614 Synaptoneurosomes were prepared based on previously published protocols ${ }^{85,86}$ with slight

615 modifications. The freshly harvested cortex tissue homogenized using dounce homogenizer

616 for 12 strokes at $4^{\circ} \mathrm{C}$ in buffer $(10 \% \mathrm{w} / \mathrm{v})$ containing $\mathrm{pH} 7.4,10 \mathrm{mM}$ 4-(2 hydroxyethyl)-1-

617 piperazineethanesulfonic acid (HEPES; Biosolve 08042359), $0.35 \mathrm{M}$ Sucrose, $1 \mathrm{mM}$

618 ethylenediaminetetraacetic acid (EDTA; VWR 0105), $0.25 \mathrm{mM}$ dithiothreitol (Thermo Fisher

619 Scientific R0861), $30 \mathrm{U} / \mathrm{ml}$ RNAse inhibitor (Life Technologies N8080119) and complete-

620 EDTA free protease inhibitor cocktail (Roche 11836170001, PhosSTOP (Roche

621 04906845001). 200ul of the total homogenate were saved for RNA extraction or western blot

622 analysis. The remaining homogenate was spun at $1000 \mathrm{~g}, 15 \mathrm{~min}$ at $4^{\circ} \mathrm{C}$ to remove the

623 nuclear and cell debris. The supernatant was sequentially passed through three $100 \mu \mathrm{m}$

624 nylon net filters (Millipore NY1H02500), followed by one $5 \mu \mathrm{m}$ filter (Millipore SMWP013000).

625 The filtrate was resuspended in 3 volumes of SNS buffer without sucrose and spun at

$6262000 \mathrm{~g}, 15 \mathrm{~min}$ at $4^{\circ} \mathrm{C}$ to collect the pellet containing synaptoneurosomes. The pellets were

627 resuspended in RIPA buffer for western blot or in qiazol reagent for RNA extraction. 
630 Total lysate and synaptoneurosomes isolated from cortex tissue of 1-month-old C57BI/6

631 mice were UV crosslinked $\left(100 \mathrm{~mJ} / \mathrm{cm}^{2}\right.$ for 2 cycles) using UV Stratalinker 2400

632 (Stratagene) and stored at $-80^{\circ} \mathrm{C}$ until use. For the total sample, cortex tissue was

633 dissociated using a cell strainer of pore size $100 \mu \mathrm{m}$ before crosslinking. We used cortex

634 from 200 mice to prepare SNS and two mice for the total cortex sample. We used a mouse

635 monoclonal antibody specific for the C-terminus of FUS (Santa Cruz) to pull down FUS

636 associated RNAs using magnetic beads. After immunoprecipitation, FUS-RNA complexes

637 were treated with MNAse in mild conditions and the 5' end of RNAs were radiolabeled with

$638 \mathrm{P}^{32}$-gamma ATP. Samples run on SDS-gel (10\% Bis Tris) were transferred to nitrocellulose

639 membrane and visualized using FLA phosphorimager. RNAs corresponding to FUS-RNA

640 complexes were purified from the nitrocellulose membrane and strand-specific paired-end

641 CLIP libraries were sequenced on HiSeq 2500 for 15 cycles.

642

643 Bioinformatic analysis of CLIP-seq data and identification of FUS targets

644 Low quality reads were filtered and adapter sequences were removed with Trim Galore!

645 (Krueger, F., TrimGalore. Retrieved February 24, 2010, from

$646 \mathrm{https}: / /$ github.com/FelixKrueger/TrimGalore). Reads were aligned to the mouse reference

647 genome (build GRCm38) using STAR version 2.4.2a ${ }^{87}$ and Ensembl gene annotations

648 (version 90). We allowed a maximum of two mismatches per read (--outFilterMismatchNmax

649 2) and removed all multimapping reads (--outFilterMultimapNmax 1). PCR duplicates were

650 removed with Picard tools version 2.18.4 ("Picard Toolkit." 2019. Broad Institute, GitHub

651 Repository. http://broadinstitute.github.io/picard/; Broad Institute). Peaks were called

652 separately on each sample with CLIPper ${ }^{52}$ using default parameters.

653 To identify regions that are specifically bound by FUS in the SNS sample but not the total

654 cortex sample, we filtered the peaks based on an MA plot. For each peak, we counted the

655 number of overlapping reads in the SNS ( $x$ ) and total cortex samples (y). M (log2 fold

656 change) and A (average log2 counts) were calculated as follows:

657

$658 M=\log 2[(x+0) /($ lib.size_ $x+0)]-\log 2[(y+0) /($ lib.size_ $y+0)]$

$659 A=[\log 2(x+0)+\log 2(y+o)] / 2$

660

661 where $0=1$ is an offset to prevent a division by 0 and lib.size_x and lib.size_y is the 662 effective library size of the two samples: the library size (number of reads mapping to the 663 peaks) multiplied by the normalization factor obtained from "calcNormFactors" using the 664 trimmed mean of $M-$ values $^{88}$ method. The $M$ and $A$ values of all CLIPper peaks identified in 
665 the SNS sample were plotted against each other (x-axis A, $y$-axis M). The plot was not 666 centered at a log2FC of 0 . Therefore, we fitted a LOESS (locally estimated scatterplot 667 smoothing) curve for normalization (loess (formula=M A, span=1/4, family="symmetric", 668 degree=1, iterations=4)). We computed the predicted $M$ values (fitted) for each $A$ value and 669 adjusted the $M$ values by the fit (adjusted $M=M-$ fitted $M$ ). After adjustment, the fitted 670 LOESS line crosses the $y$-axis at 0 with slope $=0$ in the adjusted MA-plot.

671 For ranking purposes, we computed p-values for each peak with the Bioconductor edgeR 672 package ${ }^{88}$. We computed the common dispersion of the peaks at the center of the main 673 point cloud $(-3<y<1$ in raw MA-plot) and not the tagwise dispersion because we are 674 lacking replicate information. Peak specific offsets were computed as log 675 (lib.size*norm.factors) where norm.factors are the normalization factors. The fitted M-values 676 were subtracted from the peak specific offsets to use the adjustments from the LOESS fit for 677 the statistical inference. We fit a negative binomial generalized linear model to the peak specific read counts using the adjusted offsets. We want to test for differential read counts 679 between the synaptoneurosome and total cortex sample ( group). A likelihood ratio test ${ }^{89}$ 680 was run on each peak to test for synaptoneurosome versus total cortex differences.

681 We compared the sets of peaks obtained from different p-value cutoffs (Supplementary Fig. $682 \mathrm{2g}$ ) and choose the most stringed cutoff of $1 \mathrm{e}-5$ because it showed the strongest depletion 683 of intronic peaks and strongest enrichment of exonic and 3'UTR peaks. CLIPper annotated 684 each peak to a gene and we manually inspected the assigned genes and removed wrong 685 assignments caused by overlapping gene annotations.

686 Total cortex-specific peaks (regions that are exclusively bound in the total cortex sample but 687 not the SNS sample) were computed with the same approach: the M values were computed 688 as

$689 M=\log 2\left((y+0) /\left(\operatorname{lib} . s i z e \_y+0\right)\right)-\log 2((x+0) /($ lib.size_ $x+0))$

690 and we used a p-value cutoff of 0.0029825 because that resulted in an identical number of 691 SNS-specific peaks.

692 For the over representation analysis (ORA) we applied the "goana" function from the limma $693 \mathrm{R}$ package using the gene length as covariate ${ }^{90}$. As background set, we used all genes with 694 a cpm of at least 1 in all RNA-seq samples of synaptoneurosomes from 1-month-old mice.

695 RNA motifs of length 2-8 were predicted with HOMER ${ }^{54}$. To help with the motif finding, we 696 decided to use input sequences of equal length because the lengths of the predicted peaks 697 varied a lot. We define the peak center as the median position with maximum read 698 coverage. Then, we centered a window of size 41 on the peak center of each selected peak 699 and extracted the genomic sequence. We generated background sequences for each set of 700 target sequences. A background set consists of 200,000 sequences of length 41 from 701 random locations with the same annotation as the corresponding target set (intron, exon, 3' 
702 UTR or 5' UTR). All background sequences are from regions without any read coverage in

703 the corresponding CLIP-seq sample to ensure that the background sequences are not

704 bound by FUS.

705

706

\section{RNA extraction and high-throughput sequencing (RNA-seq)}

707

Cortex tissue was isolated from 1 and/or 6-month-old Fus ${ }^{\Delta N L S /+}$ and Fus ${ }^{+++}$mice. Paired total

708 cortex $(200 \mu \mathrm{l})$ and SNS sample was obtained from a single mouse per condition using filtration protocol as previously described. Briefly, frozen total and SNS samples were mixed with Qiazol reagent following the manufacturer's recommendations and incubated at RT for $5 \mathrm{~min}$. Two hundred microliters of chloroform were added to the samples and mixed for $15 \mathrm{~s}$ and then centrifuged for $15 \mathrm{~min}\left(12,000 \mathrm{~g}, 4^{\circ} \mathrm{C}\right)$. To the upper aqueous phase collected, five hundred microliters of isopropanol and $0.8 \mu \mathrm{l}$ of glycogen was added and incubated at RT for 15 minutes. The samples were centrifuged at $10,000 \mathrm{rpm}$ for $10 \mathrm{~min}$. After centrifugation at $12,000 \mathrm{~g}$ for $15 \mathrm{~min}$, the isopropanol was removed and the pellet was washed with $1 \mathrm{ml}$ of $70 \%$ ethanol and samples were centrifuged for $5 \mathrm{~min}$ at $7500 \mathrm{~g}$. Ethanol was discarded and the RNA pellet was air-dried and dissolved in nuclease free water and further purified using the RNeasy Mini Kit including the DNAse I digestion step. The concentration and the RIN values were determined by Bioanalyzer. $150 \mathrm{ng}$ of total RNA were used for Poly A library preparation. Strand specific cDNA libraries were prepared and sequenced on lllumina NovaSeq6000 platform (2x150bp, paired end) from Eurofins Genomics, Konstanz, Germany.

\section{Bioinformatic analysis of RNA-seq data}

724 The preprocessing, gene quantification and differential gene expression analysis was 725 performed with the ARMOR workflow ${ }^{91}$. In brief, reads were quality filtered and adapters 726 were removed with Trim Galore! (Krueger, F., TrimGalore. Retrieved February 24, 2010, 727 from https://github.com/FelixKrueger/TrimGalore). For visualization purposes, reads were

728 mapped to the mouse reference genome GRCm38 with STAR version 2.4.2a ${ }^{87}$ and default 729 parameters using Ensembl gene annotations (version 90). BAM files were converted to 730 BigWig files with bedtools ${ }^{92}$. Transcript abundance estimates were computed with Salmon 731 version $0.10 .2^{93}$ and summarized to gene level with the tximeta R package ${ }^{94}$. All downstream 732 analyses were performed in $\mathrm{R}$ and the edgeR package ${ }^{88}$ was used for differential gene 733 expression analysis. We filtered the lowly expressed genes and kept all genes with a CPM 734 of at least 10/median_library_size*1e6 in 4 replicates (the size of the smallest group, here 735 the nuclear samples). Additionally, each kept gene is required to have at least 15 counts 736 across all samples. The filtered set of genes was used for the PCA plot and differential gene 737 expression analysis. 
Total RNA was reverse transcribed using Superscript III kit (Invitrogen). For qRT-PCR, 2x

741 SYBR master mix (Thermoscientific) were used and the reaction was run in Thermocycler

742 (Applied Biosystems ViiA 7) following the manufacturer's instructions.

743

Primer list

\begin{tabular}{|l|l|l|}
\hline Gene & Forward primer sequence & Reverse primer sequence \\
\hline Actin $B$ & GGTGGGTATGGGTCAGAAGGAC & GGCTGGGGTGTTGAAGGTCTC \\
\hline Camkll $\alpha$ & AATGGCAGATCGTCCACTTC & ATGAGAGGTGCCCTCAACAC \\
\hline$P s d-95$ & GTGGGCGGCGAGGATGGTGAA & CCGCCGTTTGCTGGGAATGAA \\
\hline
\end{tabular}

745

746

747

748

749

750

751

752

753

754

755

756

757

758

759

760

761

762

763

764

765

766

767

768

769

770

\section{SDS-PAGE and Western blotting}

Protein concentrations were determined using the Pierce BCA Protein Assay (Thermo Fisher Scientific) prior to SDS-PAGE. $20 \mu \mathrm{g}$ for total protein were used for western blots.

The samples were resuspended in $1 \mathrm{X}$ SDS loading buffer with $1 \mathrm{X}$ final sample reducing reagent and boiled at $95^{\circ} \mathrm{C}, 10$ mins. Samples were separated by Bolt $4-12 \%$ Bis-Tris precast gels and transferred onto nitrocellulose membranes using iBlot $\circledast$ transfer NC stacks with iBlot Dry Blotting system (Invitrogen). Membranes were blocked with buffer containing 0.05\% v/v Tween-20 (Sigma P1379) prepared in PBS (PBST) with 5\% w/v non-fat skimmed powdered milk and probed with primary antibodies (list attached) overnight at $4^{\circ} \mathrm{C}$ in PBST with $1 \% \mathrm{w} / \mathrm{v}$ milk. Following three washes with PBST, membranes were incubated with secondary HRP-conjugated goat anti mouse or rabbit AffiniPure IgG antibodies (1:5000, 1:10000, respectively) (Jackson ImmunoResearch 115-035-146 and 111-035-144, respectively) in PBST with $1 \%$ w/v milk, for 1.5 hours at RT. Membranes were washed with PBST, and the bands were visualized using Amersham Imager 600RGB (GE Healthcare Life Sciences 29083467).

\section{Transmission Electron Microscopy}

SNS pellets were prepared from cortical tissue of 1-month-old C57/BI6 mice as previously mentioned before and submitted to imaging facility at ZMB UZH. Briefly, SNS pellet prepared were re-suspended in 2X fixative (5\% Glutaraldehyde in $0.2 \mathrm{M}$ Cacodylate buffer) and fixed at RT for 30 mins. Sample was then washed twice with $0.1 \mathrm{M}$ Cacodylate buffer before embedding into $2 \%$ Agar Nobile. Post-fixation was performed with $1 \%$ Osmium 1 hour on ice, washed three times with $\mathrm{ddH}_{2} \mathrm{O}$, dehydrated with $70 \%$ ethanol for 20 mins, followed by $80 \%$ ethanol for 20 mins, $100 \%$ for 30 mins and finally Propylene for 30 mins. 
771 Propylene: Epon Araldite at 1:1 were added overnight followed by addition of Epon Araldite

772 for 1 hour at RT. Sample was then embedded via 28 hours incubation at $60^{\circ} \mathrm{C}$. The resulting

773 block was then cut into $60 \mathrm{~nm}$ ultrathin sections using ultramicrotome. Ribbons of sections

774 were then put onto TEM grid and imaged on TEM - FEI CM100 electron microscope

775 (modify).

776

777 Confocal image acquisition and analysis

778 Confocal images were acquired on a Leica SP8 Falcon microscope using 63X (NA 1.4) with

779 a zoom power of 3 . Images were acquired at a $2048 \times 2048$ pixel size, yielding to a 30.05

$780 \mathrm{~nm} /$ pixel resolution. To quantify the density of synaptic markers, images were acquired in

781 CA1 region in the apical dendrite area, $\sim 50 \mu \mathrm{m}$ from the soma, at the bifurcation of the

782 apical dendrite of pyramidal cells, using the same parameters for both genotypes. Images

783 were acquired from top to bottom with a Z step size of $500 \mathrm{~nm}$. Images were deconvoluted

784 using Huygens Professional software (Scientific Volume Imaging). Images were then

785 analyzed as described previously ${ }^{84}$. Briefly, stacks were analyzed using the built-in particle

786 analysis function in $\mathrm{Fiji}^{95}$. The size of the particles was defined according to previously

787 published studies ${ }^{80,96,97}$. To assess the number of clusters, images were thresholded (same

788 threshold per marker and experiment), and a binary mask was generated. A low size

789 threshold of $0.01 \mu \mathrm{m}$ diameter and high pass threshold of $1 \mu \mathrm{m}$ diameter was applied. Top

790 and bottom stacks were removed from the analysis to only keep the 40 middle stacks. For

791 the analysis, the number of clusters per $40 \mathrm{z}$ stacks was summed and normalized by the

792 volume imaged $(75153.8 \mu \mathrm{m} 3)$. The density was normalized by the control group. The

793 densities were compared by $t$ test for 1- and 6-month-old mice. GluN1 synaptic localization

794 was analyzed by counting the number of colocalized GluN1 clusters with Synapsin 1.

795 Colocalization clusters were generated using ImageJ plugin colocalization highlighter. The

796 default parameters were applied to quantify the colocalization. The number of colocalized

797 clusters were quantified using the built-in particle analysis function in Fiji ${ }^{95}$.

798

799 Synaptic density and composition imaging and analysis of primary neuronal culture

800 Imaging and quantification were performed as previously reported ${ }^{98}$. Briefly, synaptic density

801 and synapse composition was assayed in 22 DIV neuronal cell cultures. Cultures were fixed

802 in cold 4\% PFA with 4\% sucrose for 20 minutes at RT. Primary antibodies were incubated

803 overnight at $4^{\circ} \mathrm{C}$. secondary antibodies were incubated for $3 \mathrm{~h}$ at RT. Hippocampal primary

804 culture: pyramidal cells were selected based on their morphology and confocal images were

805 acquired on a Leica SP8 Falcon microscope using 63X (NA 1.4) with a zoom power of 3 and

806 analyzed with Fiji software. After deconvolution (huygens professional), images were 
807 subsequently thresholded, and subsequent analyses were performed by an investigator

808 blind to cell culture treatment.

809

810

811

812

813

814

815

816

817

818

819

820

821

822

823

824

Antibody list

\begin{tabular}{|l|l|l|l|l|}
\hline Antibody & Species, Source & $\begin{array}{l}\text { STORM } \\
\text { dilution }\end{array}$ & $\begin{array}{l}\text { Confocal } \\
\text { dilution }\end{array}$ & $\begin{array}{l}\text { Western blot } \\
\text { dilution }\end{array}$ \\
\hline FUS & Rb, A300-293A, Bethyl & & $1: 500$ & $1: 1000$ \\
\hline FUS & Rb, A300-294A, Bethyl & & $1: 1000$ \\
\hline FUS & Ms, 4H11, Santa Cruz & $1: 200$ & & \\
\hline PSD-95 & Ms, Invitrogen & $1: 200$ & $1: 1000$ & $1: 1000$ \\
\hline P-CAMKIla & $\begin{array}{l}\text { Ms, D21E4, Cell } \\
\text { signaling }\end{array}$ & $1: 500$ & $1: 500$ & $1: 1000$ \\
\hline PNF & Ms, SMl31, Covance & & $1: 1000$ & \\
\hline Spinophilin & Rb, Synaptic Systems & $1: 500$ & & \\
\hline Synapsin 1 & Ms, Synaptic Systems & $1: 200$ & $1: 500$ & \\
\hline GluA1 & Rb, Sigma Aldrich & $1: 200$ & $1: 200$ & $1: 1000$ \\
\hline GluN1 & Ms, Covance & & $1: 500$ & \\
\hline GluN2B & Rb, Sigma Aldrich & $1: 500$ & & $1: 2000$ \\
\hline Bassoon & Gp, Synaptic Systems & $1: 500$ & $1: 500$ & \\
\hline GRP78 BiP (ER) & Rb, Abcam & $1: 200$ & & \\
\hline
\end{tabular}




\begin{tabular}{|l|l|l|l|l|}
\hline & & & & \\
\hline MAP2 & Ms, Sigma Aldrich & & $1: 1000$ & \\
\hline SYP & Ms, Santa cruz & & & $1: 200$ \\
\hline GABAA/alpha1 & Gp, Synaptic Systems & & $1: 500$ & \\
\hline GABAA/alpha3 & Rb, Synaptic Systems & & $1: 500$ & \\
\hline Gephyrin & Ms, Synaptic Systems & & $1: 500$ & \\
\hline Vgat & Gp, Synaptic Systems & & $1: 500$ & \\
\hline 及-Actin & Ms, Sigma & & & $1: 5000$ \\
\hline SNAP25 & Gp, Synaptic Systems & & $1: 500$ & $1: 1000$ \\
\hline
\end{tabular}

825

Author Contribution

827 Conceptualization of the study was carried by S.S., K.M.H., and M.P.. S.S. performed 828 synaptosome isolation, CLIP-seq sample preparation and RNA-seq sample preparation. 829 K.M.H. analyzed the data from CLIP-seq and RNA-seq. S.S., K.M.H., M.D.R. and M.P. 830 developed the strategy to analyze the sequencing data. E.T., M.H.P., M.P.B., J.W., and P.S. 831 provided experimental support for the experiments. L.D. provided the mouse model and 832 input on the study. P.D.R. performed immunostaining and image analyses including 833 confocal, STED and dSTORM. S.S., K.M.H, E.T, P.D.R and M.P wrote and edited the 834 manuscript. M.D.R, P.D.R and M.P. provided supervision. M.P directed the entire study. All 835 authors read, edited, and approved the final manuscript.

836

\section{Acknowledgments}

838 We gratefully acknowledge the support of the National Centre for Competence in Research (NCCR) RNA \& Disease funded by the Swiss National Science Foundation. SSMK was supported by Swiss Government Excellence Scholarships for Foreign Scholars. The authors would like to thank Prof. Adriano Aguzzi and Dr. Claudia Scheckel for helpful discussions and Dr. Dorothee Dormann for critical comments on the manuscript. We thank Gery

843 Barmettler and Dr. José María Mateos from ZMB UZH for technical help with TEM. We also 844 thank Catharina Aquino and Lucy Poveda from FGCZ for discussions and technical help on 845 CLIP library preparation and sequencing. 
bioRxiv preprint doi: https://doi.org/10.1101/2020.06.10.136010; this version posted June 10, 2020. The copyright holder for this preprint (which was not certified by peer review) is the author/funder, who has granted bioRxiv a license to display the preprint in perpetuity. It is made available under aCC-BY-NC-ND 4.0 International license.

850

851

852

853

854

855

856 


\section{References}

858

859 1. Lagier-Tourenne, C., Polymenidou, M. \& Cleveland, D. W. TDP-43 and FUS/TLS: Emerging

860 roles in RNA processing and neurodegeneration. Hum. Mol. Genet. 19, 46-64 (2010).

861 2. Andersson, M. K. et al. The multifunctional FUS, EWS and TAF15 proto-oncoproteins show

862 cell type-specific expression patterns and involvement in cell spreading and stress response.

863 BMC Cell Biol. (2008). doi:10.1186/1471-2121-9-37

864 3. Dormann, D. et al. ALS-associated fused in sarcoma (FUS) mutations disrupt transportin-

865 mediated nuclear import. EMBO J. (2010). doi:10.1038/emboj.2010.143

866 4. Ederle, H. et al. Nuclear egress of TDP-43 and FUS occurs independently of Exportin-

867 1/CRM1. Sci. Rep. (2018). doi:10.1038/s41598-018-25007-5

868 5. Hock, E. M. et al. Hypertonic Stress Causes Cytoplasmic Translocation of Neuronal, but Not

869 Astrocytic, FUS due to Impaired Transportin Function. Cell Rep. (2018).

870 doi:10.1016/j.celrep.2018.06.094

871 6. Kwiatkowski, T. J. et al. Mutations in the FUS/TLS gene on chromosome 16 cause familial

872 amyotrophic lateral sclerosis. Science (80-. ). 323, 1205-1208 (2009).

873 7. Vance, C. et al. Mutations in FUS, an RNA processing protein, cause familial amyotrophic

874 lateral sclerosis type 6. Science (80-. ). (2009). doi:10.1126/science.1165942

875 8. Neumann, M. et al. A new subtype of frontotemporal lobar degeneration with FUS pathology.

$876 \quad$ Brain 132, 2922-2931 (2009).

877 9. Lee, B. J. et al. Rules for Nuclear Localization Sequence Recognition by Karyopherinß2. Cell

878 (2006). doi:10.1016/j.cell.2006.05.049

879 10. Mackenzie, I. R. A., Rademakers, R. \& Neumann, M. TDP-43 and FUS in amyotrophic lateral

11. Sama, R. R. K. et al. FUS/TLS assembles into stress granules and is a prosurvival factor

12. Murakami, T. et al. ALS/FTD Mutation-Induced Phase Transition of FUS Liquid Droplets and and Arginine Methylation. Cell (2018). doi:10.1016/j.cell.2018.03.004 Methylation of Arginine Cation-m Interactions. Cell (2018). doi:10.1016/j.cell.2018.03.056

18. Maharana, S. et al. RNA buffers the phase separation behavior of prion-like RNA binding 
897

898

899

900

901

902

903

904

905

906

907

908

909

910

911

912

913

914

915

916

917

918

919

920

921

922

923

924

925

926

927

928

929

930

931

932

933

934

935

936

proteins. Science (80-. ). (2018). doi:10.1126/science.aar7366

19. Langdon, E. M. et al. mRNA structure determines specificity of a polyQ-driven phase separation. Science (80-. ). (2018). doi:10.1126/science.aar7432

20. Fujii, R. et al. The RNA binding protein TLS is translocated to dendritic spines by mGluR5 activation and regulates spine morphology. Curr. Biol. (2005). doi:10.1016/j.cub.2005.01.058

21. Biever, A., Donlin-Asp, P. G. \& Schuman, E. M. Local translation in neuronal processes. Current Opinion in Neurobiology (2019). doi:10.1016/j.conb.2019.02.008

22. Lagier-Tourenne, C. et al. Divergent roles of ALS-linked proteins FUS/TLS and TDP-43 intersect in processing long pre-mRNAs. Nat. Neurosci. 15, 1488-1497 (2012).

23. Udagawa, T. et al. FUS regulates AMPA receptor function and FTLD/ALS-associated behaviour via GluA1 mRNA stabilization. Nat. Commun. 6, (2015).

24. Yokoi, S. et al. 3'UTR Length-Dependent Control of SynGAP Isoform $a 2$ mRNA by FUS and ELAV-like Proteins Promotes Dendritic Spine Maturation and Cognitive Function. Cell Rep. 20, 3071-3084 (2017).

25. Schoen, M. et al. Super-resolution microscopy reveals presynaptic localization of the ALS/FTD related protein FUS in hippocampal neurons. Front. Cell. Neurosci. 9, 1-16 (2016).

26. So, E. et al. Mitochondrial abnormalities and disruption of the neuromuscular junction precede the clinical phenotype and motor neuron loss in hFUSWT transgenic mice. Hum. Mol. Genet. 27, 463-474 (2018).

27. Deshpande, D. et al. Synaptic FUS localization during motoneuron development and its accumulation in human ALS synapses. Front. Cell. Neurosci. 13, 1-17 (2019).

28. Aoki, N. et al. Localization of fused in sarcoma (FUS) protein to the post-synaptic density in the brain. Acta Neuropathol. 124, 383-394 (2012).

29. Hicks, G. G. et al. Fus deficiency in mice results in defective B-lymphocyte development and activation, high levels of chromosomal instability and perinatal death. Nat. Genet. (2000). doi:10.1038/72842

30. Kino, Y. et al. FUS/TLS deficiency causes behavioral and pathological abnormalities distinct from amyotrophic lateral sclerosis. Acta Neuropathol. Commun. 3, 24 (2015).

31. López-Erauskin, J. et al. ALS/FTD-Linked Mutation in FUS Suppresses Intra-axonal Protein Synthesis and Drives Disease Without Nuclear Loss-of-Function of FUS. Neuron 100, 816830.e7 (2018).

32. Fogarty, M. J. Driven to decay: Excitability and synaptic abnormalities in amyotrophic lateral sclerosis. Brain Research Bulletin (2018). doi:10.1016/j.brainresbull.2018.05.023

33. Starr, A. \& Sattler, R. Synaptic dysfunction and altered excitability in C9ORF72 ALS/FTD. Brain Research (2018). doi:10.1016/j.brainres.2018.02.011

34. Henstridge, C. M. et al. Synapse loss in the prefrontal cortex is associated with cognitive decline in amyotrophic lateral sclerosis. Acta Neuropathol. (2018). doi:10.1007/s00401-0171797-4

35. Sephton, C. F. \& Yu, G. The function of RNA-binding proteins at the synapse: Implications for neurodegeneration. Cellular and Molecular Life Sciences (2015). doi:10.1007/s00018-015- 
937

938

939

940

941

942

943

944

945

946

947

948

949

950

951

952

953

954

955

956

957

958

959

960

961

962

963

964

965

966

967

968

969

970

971

972

973

974

975

976

1943-x

36. Selkoe, D. J. Alzheimer's disease is a synaptic failure. Science (2002). doi:10.1126/science.1074069

37. Hoell, J. I. et al. RNA targets of wild-type and mutant FET family proteins. Nat. Struct. Mol. Biol. (2011). doi:10.1038/nsmb.2163

38. Rogelj, B. et al. Widespread binding of FUS along nascent RNA regulates alternative splicing in the brain. Sci. Rep. 2, 1-10 (2012).

39. Ishigaki, S. et al. Position-dependent FUS-RNA interactions regulate alternative splicing events and transcriptions. Sci. Rep. 2, 1-8 (2012).

40. Masuda, A. et al. Position-specific binding of FUS to nascent RNA regulates mRNA length. Genes Dev. 29, 1045-1057 (2015).

41. Nakaya, T., Alexiou, P., Maragkakis, M., Chang, A. \& Mourelatos, Z. FUS regulates genes coding for RNA-binding proteins in neurons by binding to their highly conserved introns. RNA (2013). doi:10.1261/rna.037804.112

42. Loughlin, F. E. et al. The Solution Structure of FUS Bound to RNA Reveals a Bipartite Mode of RNA Recognition with Both Sequence and Shape Specificity. Mol. Cell (2019). doi:10.1016/j.molcel.2018.11.012

43. Scekic-Zahirovic, J. et al. Toxic gain of function from mutant FUS protein is crucial to trigger cell autonomous motor neuron loss. EMBO J. 35, 1077-97 (2016).

44. Gerth, F. et al. Intersectin associates with synapsin and regulates its nanoscale localization and function. Proc. Natl. Acad. Sci. U. S. A. (2017). doi:10.1073/pnas.1715341114

45. Nishimune, H., Badawi, Y., Mori, S. \& Shigemoto, K. Dual-color STED microscopy reveals a sandwich structure of Bassoon and Piccolo in active zones of adult and aged mice. Sci. Rep. (2016). doi:10.1038/srep27935

46. Fukata, Y. et al. Local palmitoylation cycles define activity-regulated postsynaptic subdomains. J. Cell Biol. (2013). doi:10.1083/jcb.201302071

47. Wang, W. Y. et al. Interaction of FUS and HDAC1 regulates DNA damage response and repair in neurons. Nat. Neurosci. (2013). doi:10.1038/nn.3514

48. Rulten, S. L. et al. PARP-1 dependent recruitment of the amyotrophic lateral sclerosisassociated protein FUS/TLS to sites of oxidative DNA damage. Nucleic Acids Res. (2014). doi:10.1093/nar/gkt835

49. Tan, A. Y. \& Manley, J. L. TLS Inhibits RNA Polymerase III Transcription. Mol. Cell. Biol. (2010). doi:10.1128/mcb.00884-09

50. Schwartz, J. C. et al. FUS binds the CTD of RNA polymerase II and regulates its phosphorylation at Ser2. Genes Dev. (2012). doi:10.1101/gad.204602.112

51. Polymenidou, M. et al. Long pre-mRNA depletion and RNA missplicing contribute to neuronal vulnerability from loss of TDP-43. Nat. Neurosci. (2011). doi:10.1038/nn.2779

52. Lovci, M. T. et al. Rbfox proteins regulate alternative mRNA splicing through evolutionarily conserved RNA bridges. Nat. Struct. Mol. Biol. 20, 1434-1442 (2013).

53. Wang, T., Xie, Y. \& Xiao, G. dCLIP: A computational approach for comparative CLIP-seq 
982

983

984

985

986

987

988

989

990

991

992

993

994

995

996

997

998

999

1000

1001

1002

1003

1004

1005

1006

1007

1008

1009

1010

1011

1012

1013

1014

1015

1016

analyses. Genome Biol. (2014). doi:10.1186/gb-2014-15-1-r11

54. Heinz, S. et al. Simple Combinations of Lineage-Determining Transcription Factors Prime cisRegulatory Elements Required for Macrophage and B Cell Identities. Mol. Cell (2010). doi:10.1016/j.molcel.2010.05.004

55. Scekic Zahirovic, J. et al. Toxic gain of function from mutant FUS protein is crucial to trigger cell autonomous motor neuron loss . EMBO J. (2016). doi:10.15252/embj.201592559

56. Preston, A. R. \& Eichenbaum, H. Interplay of hippocampus and prefrontal cortex in memory. Current Biology (2013). doi:10.1016/j.cub.2013.05.041

57. Somogyi, P., Tamás, G., Lujan, R. \& Buhl, E. H. Salient features of synaptic organisation in the cerebral cortex. in Brain Research Reviews (1998). doi:10.1016/S0165-0173(97)00061-1

58. Zhu, F. et al. Architecture of the Mouse Brain Synaptome. Neuron (2018). doi:10.1016/j.neuron.2018.07.007

59. Contreras, A., Hines, D. J. \& Hines, R. M. Molecular specialization of GABAergic synapses on the soma and axon in cortical and hippocampal circuit function and dysfunction. Frontiers in Molecular Neuroscience (2019). doi:10.3389/fnmol.2019.00154

60. Distler et al. Proteomic Analysis of Brain Region and Sex-Specific Synaptic Protein Expression in the Adult Mouse Brain. Cells 9, 313 (2020).

61. Irfan, M. et al. SNAP-25 isoforms differentially regulate synaptic transmission and long-term synaptic plasticity at central synapses. Sci. Rep. (2019). doi:10.1038/s41598-019-42833-3

62. Chaudhry, F. A. et al. The vesicular GABA transporter, VGAT, localizes to synaptic vesicles in sets of glycinergic as well as GABAergic neurons. J. Neurosci. (1998). doi:10.1523/jneurosci.18-23-09733.1998

63. Mcllhinney, R. A. J. et al. Assembly of N-methyl-D-aspartate (NMDA) receptors. in Biochemical Society Transactions (2003). doi:10.1042/BST0310865

64. Diering, G. H. \& Huganir, R. L. The AMPA Receptor Code of Synaptic Plasticity. Neuron (2018). doi:10.1016/j.neuron.2018.10.018

65. Sigel, E. \& Steinmann, M. E. Structure, function, and modulation of GABAA receptors. Journal of Biological Chemistry (2012). doi:10.1074/jbc.R112.386664

66. Choii, G. \& Ko, J. Gephyrin: a central GABAergic synapse organizer. Experimental \& molecular medicine (2015). doi:10.1038/emm.2015.5

67. Brüning, F. et al. Sleep-wake cycles drive daily dynamics of synaptic phosphorylation. Science (80-. ). 366, (2019).

68. Holt, C. E., Martin, K. C. \& Schuman, E. M. Local translation in neurons: visualization and function. Nat. Struct. Mol. Biol. 26, 557-566 (2019).

69. Kosik, K. S. Life at Low Copy Number: How Dendrites Manage with So Few mRNAs. Neuron (2016). doi:10.1016/j.neuron.2016.11.002

70. Cajigas, I. J. et al. The Local Transcriptome in the Synaptic Neuropil Revealed by Deep Sequencing and High-Resolution Imaging. Neuron 74, 453-466 (2012).

71. Fritschy, J. M \& Mohler, H. GABAA receptor heterogeneity in the adult rat brain: Differential regional and cellular distribution of seven major subunits. J. Comp. Neurol. (1995). 
1017

1018

1019

1020

1021

1022

1023

1024

1025

1026

1027

1028

1029

1030

1031

1032

1033

1034

1035

1036

1037

1038

1039

1040

1041

1042

1043

1044

1045

1046

1047

1048

1049

1050

1051

1052

1053

1054

1055

1056

doi:10.1002/cne.903590111

72. Dias, R. et al. Evidence for a significant role of a3-containing GABAA receptors in mediating the anxiolytic effects of benzodiazepines. J. Neurosci. (2005). doi:10.1523/JNEUROSCI.116605.2005

73. Smith, K. S., Engin, E., Meloni, E. G. \& Rudolph, U. Benzodiazepine-induced anxiolysis and reduction of conditioned fear are mediated by distinct GABAA receptor subtypes in mice. Neuropharmacology (2012). doi:10.1016/j.neuropharm.2012.03.001

74. Fischer, B. D. et al. Contribution of GABAA receptors containing $\alpha 3$ subunits to the therapeutic-related and side effects of benzodiazepine-type drugs in monkeys. Psychopharmacology (Berl). (2011). doi:10.1007/s00213-010-2142-y

75. Husi, H., Ward, M. A., Choudhary, J. S., Blackstock, W. P. \& Grant, S. G. N. Proteomic analysis of NMDA receptor-adhesion protein signaling complexes. Nat. Neurosci. 3, 661-669 (2000).

76. Tafoya, L. C. R. et al. Expression and function of SNAP-25 as a universal SNARE component in GABAergic neurons. J. Neurosci. (2006). doi:10.1523/JNEUROSCI.1866-06.2006

77. Wojcik, S. M. et al. A Shared Vesicular Carrier Allows Synaptic Corelease of GABA and Glycine. Neuron (2006). doi:10.1016/j.neuron.2006.04.016

78. Mukherjee, J. et al. The residence time of GABA ARs at inhibitory synapses is determined by direct binding of the receptor a1 subunit to gephyrin. J. Neurosci. (2011). doi:10.1523/JNEUROSCI.2001-11.2011

79. Tretter, V. et al. Molecular basis of the $\mathrm{y}$-aminobutyric acid a receptor $\alpha 3$ subunit interaction with the clustering protein gephyrin. J. Biol. Chem. (2011). doi:10.1074/jbc.M111.291336

80. Specht, C. G. et al. Quantitative nanoscopy of inhibitory synapses: Counting gephyrin molecules and receptor binding sites. Neuron (2013). doi:10.1016/j.neuron.2013.05.013

81. Schieweck, R. \& Kiebler, M. A. Posttranscriptional gene regulation of the GABA receptor to control neuronal inhibition. Front. Mol. Neurosci. (2019). doi:10.3389/fnmol.2019.00152

82. He, Q. \& Ge, W. The tandem Agenet domain of fragile $X$ mental retardation protein interacts with FUS. Sci. Rep. (2017). doi:10.1038/s41598-017-01175-8

83. Heraud-Farlow, J. E. \& Kiebler, M. A. The multifunctional Staufen proteins: Conserved roles from neurogenesis to synaptic plasticity. Trends in Neurosciences (2014). doi:10.1016/j.tins.2014.05.009

84. Rossi, P. De, Nomura, T., Andrew, R. J. \& Nicholson, D. A. Neuronal BIN1 Regulates Presynaptic Neurotransmitter Release and Memory Article Neuronal BIN1 Regulates Presynaptic Neurotransmitter Release and Memory Consolidation. CellReports 30, 35203535.e7 (2020).

85. Williams, C. et al. Transcriptome analysis of synaptoneurosomes identifies neuroplasticity genes overexpressed in incipient Alzheimer's disease. PLoS One (2009). doi:10.1371/journal.pone.0004936

86. Muddashetty, R. S., Kelić, S., Gross, C., Xu, M. \& Bassell, G. J. Dysregulated metabotropic glutamate receptor-dependent translation of AMPA receptor and postsynaptic density-95 
1057

1058

1059

1060

1061

1062

1063

1064

1065

1066

1067

1068

1069

1070

1071

1072

1073

1074

1075

1076

1077

1078

1079

1080

1081

1082

1083

1084

1085

1086

1087

1088

1089

1090

1091

1092

1093

1094

1095

1096

1097

mRNAs at synapses in a mouse model of fragile X syndrome. J. Neurosci. (2007). doi:10.1523/JNEUROSCI.0937-07.2007

87. Dobin, A. et al. STAR: Ultrafast universal RNA-seq aligner. Bioinformatics (2013). doi:10.1093/bioinformatics/bts635

88. Robinson, M. D. \& Oshlack, A. A scaling normalization method for differential expression analysis of RNA-seq data. Genome Biol. (2010). doi:10.1186/gb-2010-11-3-r25

89. McCarthy, D. J., Chen, Y. \& Smyth, G. K. Differential expression analysis of multifactor RNASeq experiments with respect to biological variation. Nucleic Acids Res. (2012). doi:10.1093/nar/gks042

90. Young, M. D., Wakefield, M. J., Smyth, G. K. \& Oshlack, A. Gene ontology analysis for RNAseq: accounting for selection bias. Genome Biol. (2010). doi:10.1186/gb-2010-11-2-r14

91. Orjuela, S., Huang, R., Hembach, K. M., Robinson, M. D. \& Soneson, C. ARMOR: An automated reproducible modular workflow for preprocessing and differential analysis of RNAseq data. G3 Genes, Genomes, Genet. (2019). doi:10.1534/g3.119.400185

92. Quinlan, A. R. \& Hall, I. M. BEDTools: A flexible suite of utilities for comparing genomic features. Bioinformatics (2010). doi:10.1093/bioinformatics/btq033

93. Patro, R., Duggal, G., Love, M. I., Irizarry, R. A. \& Kingsford, C. Salmon provides fast and bias-aware quantification of transcript expression. Nat. Methods (2017).

doi:10.1038/nmeth.4197

94. Love, M. I. et al. Tximeta: reference sequence checksums for provenance identification in RNA-seq. bioRxiv (2019). doi:10.1101/777888

95. Rueden, C. T. et al. ImageJ2: Image J for the next generation of scientific image data. BMC Bioinformatics (2017). doi:10.1186/s12859-017-1934-z

96. MacGillavry, H. D., Song, Y., Raghavachari, S. \& Blanpied, T. A. Nanoscale scaffolding domains within the postsynaptic density concentrate synaptic ampa receptors. Neuron (2013). doi:10.1016/j.neuron.2013.03.009

97. Nair, D. et al. Super-resolution imaging reveals that AMPA receptors inside synapses are dynamically organized in nanodomains regulated by PSD95. J. Neurosci. (2013). doi:10.1523/JNEUROSCI.2381-12.2013

98. De Rossi, P. et al. Predominant expression of Alzheimer's disease-associated BIN1 in mature oligodendrocytes and localization to white matter tracts. Mol. Neurodegener. (2016). doi:10.1186/s13024-016-0124-1

99. Noya, S. B. et al. The forebrain synaptic transcriptome is organized by clocks but its proteome is driven by sleep. Science (80-. ). 366, (2019).

\section{Figure legends}

Fig. 1 FUS is enriched at the presynaptic compartment 
1098 (a) Confocal images showing the distribution of FUS (green) in the pyramidal layer of the

1099

1100

1101

1102

1103

1104

1105

1106

1107

1108

1109

1110

1111

1112

1113

1114

1115

1116

1117

1118

1119

1120

1121

1122

1123

1124

1125

1126

1127

1128

1129

1130

1131

1132

1133

1134 retrosplenial cortical area along with MAP2 (blue) and PNF (magenta). Left panel shows the overview and the right panel the zoomed in area labelled with the red box on the left panel. (b) Similar confocal images showing FUS (green) along with PSD95 (orange) and Synapsin 1 (Syn, blue. (c) Synaptic localization of FUS was assessed by STED microscopy using excitatory (PSD95) and inhibitory (VGAT) markers for synapses. $60 \mu \mathrm{m}$ brain sections were analyzed and distance between FUS and the synaptic markers was analyzed using Imaris. (d) Bar graph representing the percentage of synapses within $200 \mathrm{~nm}$ of FUS clusters and showing an enrichment of FUS at the excitatory synapses. (e) dSTORM was used to explore more precisely the FUS localization within the synapse, using primary culture. Bassoon and Synapsin 1 (Syn) were used to label the presynaptic compartment and GluN1, GluA1 and PSD95 were used to label the postsynapse. Spinophilin (Spino) was used to label the spines. (f) Bar graph representing the percentage of FUS localized within 100nm from presynaptic or postsynaptic markers. (g) Bar graph representing the distribution of FUS in the synapse. (h) Schematic summarizing the FUS localization within the synapse. Graph bar showing mean + SD. ${ }^{*} p>0.05,{ }^{* *} p>0.01,{ }^{* * *} p>0.001,{ }^{* * * *} p>0.000$.

Fig. 2 CLIP-seq on cortical synaptoneurosomes identified FUS-associated pre- and postsynaptic RNAs

(a) Electron microscopic images of synaptoneurosomes (SNS) from mouse cortex showing intact pre- and postsynaptic compartments. (b) Western blot of synaptic proteins (PSD95, pCamKII), nuclear protein (Lamin B1) and FUS in total and SNS. (c) qPCR shows enrichment of PSD95, CamKII mRNAs in SNS. (d) Autoradiograph of FUS-RNA complexes immunoprecipitated from total homogenate and SNS and trimmed by different concentrations of micrococcal nuclease (MNase). (e) MA-plot of CLIPper peaks predicted in the SNS CLIP-seq sample. logCPM is the average log2CPM of each peak in the total cortex and SNS sample and logFC is the log2 fold-change between the number of reads in the SNS and total cortex sample. (f) Same MA-plot as E showing the selected, SNS specific peaks ( $p$-value cutoff of 1e-05) in red. (g) Barplot with the percentage of SNS and total cortex specific peaks located in exons, 5'UTRs, 3'UTRs or introns. FUS binding in Grin1 (h), Gabra1 (i) in total cortex (green) and SNS (blue). (j) Schematic with the cellular localization and function of some of the selected FUS targets.

Fig. 3 Increased synaptic FUS localization in Fus $^{\Delta N L S /+}$ mice affect GABAergic synapses (a) Schematic showing specificity of antibodies used for western blot against protein domains of FUS. (b) Western blot of total FUS, full length FUS and actin in 
1135 synaptoneurosomes isolated from $\mathrm{Fus}^{+/+}$and $\mathrm{Fus}^{\Delta \mathrm{NLS/+}}$ mice at 6 months of age. (c)

1136 Quantification of total FUS and full length FUS levels in synaptoneurosomes from $\mathrm{Fus}^{+/+}$and

$1137 F_{U s^{\Delta N L S /+}}$ at 6 months of age. (d) Confocal images of the hippocampal CA1 area from 6-

1138 month-old mice showing higher level of FUS in the dendritic tree and synaptic compartment

1139 in FUS ${ }^{\triangle N L S /+}$ mouse-model. On the top, low magnification pictures show the dendritic area of

1140 pyramidal cells stained with FUS (green), MAP2 (dendritic marker, magenta), Synapsin 1

1141 (Syn, Synaptic marker, Cyan) and DAPI (Blue). Red box indicates the area imaged in the

1142 high magnification images below. (e) Higher magnification equivalent to the area highlighted

1143 in red in (d). (f) Representative images of staining using synaptic markers Synapsin 1,

1144 VGAT, GABA $\mathrm{A} 3$ and GluN1 in $\mathrm{Fus}^{+/+}$and $\mathrm{Fus}^{\Delta N L S /+}$ at 1 and 6 months of age. Images were

1145 generated with Imaris and display volume view used for quantification with statistically coded

1146 surface area. Density and cluster area were analyzed. (g) Graph bar representation of the 1147 synaptic density of Synapsin 1, VGAT, GABA $\alpha$ a 3 and GluN1 from Fus ${ }^{+/+}$and Fus ${ }^{\Delta N L S /+}$ at 1

1148 and 6 months of age. Graph bar showing mean + SD. ${ }^{*} p<0.05$. Graphs are extracted from

1149 the same analysis shown in Supplementary Fig. 3e-f. The statistical analysis can be found

1150 in Table 2. (h) Colocalization analysis of GluN1 with Synapsin 1 to identify synaptic NMDAR

1151 and extrasynaptic NMDAR. Results were normalized by the control of each group. Graph

1152 bar showing mean $+S D$. ${ }^{*} p<0.05$. (i) Box and Whiskers representation of the average 1153 cluster area for each marker (Synapsin1, VGAT, GABA ${ }_{A} \alpha 3$ and GluN1) from 1-month and 61154 month-old Fus ${ }^{++}$and Fus ${ }^{\Delta N L S /+}$ mice. Box showing Min to Max, ${ }^{*} p<0.05{ }^{* *} p<0.01$. Graphs are 1155 extracted from the same analysis shown in Supplementary Fig. 3f-i. The statistical analysis 1156 can be found in Table 3.

\section{Fig. 4 Age-dependent alterations in the synaptic RNA profile of $F u s^{\Delta N L S /+}$ mouse cortex}

1159

(a) Outline of the RNA-seq experiment. (b) Heatmap from the set of up-and downregulated genes in SNS of Fus ${ }^{\Delta N L S /+}$ at 6-months compared to Fus ${ }^{+/+}$. Genes are on the rows and the different samples on the columns. The color scale indicates the log2FC between the CPM of each sample and mean CPM of the corresponding $\mathrm{Fus}^{+/+}$samples at each time point [sample logCPM - mean (logCPM of $\mathrm{Fus}^{+/+}$samples)]. (c) Volcano plots showing the log2 fold change of each gene and the corresponding minus log10 (FDR) of the differential gene expression analysis comparing $\mathrm{Fus}^{\mathrm{LNLS/+}}$ SNS to $\mathrm{Fus}^{+/+}$SNS at 1 month (left panel) and 6 months of age (right panel). The horizontal line marks the significance threshold of 0.05 . Significantly downregulated genes are highlighted in green, upregulated genes in purple and all FUS targets identified in the CLIP-seq data in blue. (d) Venn diagram of the sets of significantly up- and downregulated genes (SNS of $F u s^{\Delta N L S /+}$ vs. Fus ${ }^{+/+}$at 6 months of age) and the SNS FUS target genes identified by our FUS CLIP-seq. (e) Schematic of the cellular 
1172 of age.

1173

1174

1175

1176

1177

1178

1179

1180

1181

1182

1183

1184

1185

1186

1187

1188

1189

1190

1191

1192

1193

1194

1195

1196

1197

1198

1199

1200

1201

1202

1203

1204

1205

1206

\section{Table 1: FUS binds GU-rich sequences at the synapse}

Predicted sequence motifs (HOMER) in windows of size 41 centered on the position with maximum coverage in each peak. Each set of target sequences has a corresponding background set with 200,000 sequences without any CLIP-seq read coverage (they are not bound by FUS). Note: These are all motifs that were not marked as possible false positives by HOMER and that occur in more than $1 \%$ of the target sequences.

\section{Table 2. Statistical analysis of synaptic density}

The table reports statistical analysis of density of the synaptic markers analyzed from a minimum of 2 images from at least 4 animals per genotype $\left(F u s^{+/+}\right.$and $\left.F u s^{\Delta N L S /+}\right)$ at 1 and 6 months of age. Unpaired t-test statistics, p-values, specific t-distribution (t), degrees of freedom (DF) and sample size are listed.

\section{Table 3. Statistical analysis of synaptic cluster area}

The table reports statistical analysis of area of the synaptic markers analyzed from a minimum of 2 images from at least 4 animals per genotype $\left(F u s^{+/+}\right.$and $\left.F u s^{\Delta N L S /+}\right)$ at 1 and 6 months of age. Unpaired t-test statistics, $p$-values, specific t-distribution (t), degrees of freedom (DF) and sample size are listed.

\section{Supplemental Figures titles and legends}

\section{Supplementary Fig. 1 FUS is enriched at the presynaptic compartment}

(a) Confocal images showing the distribution of FUS (green) in the molecular layer of the CA1 hippocampal area along with MAP2 (blue) and PNF (magenta). Left panel shows the overview and the right panel, the zoomed in area labelled with the red box on the left panel. (b) Similar confocal images showing FUS (green) along with PSD95 (orange) and Synapsin 1 (Syn, blue). (c) Schematic of the workflow for distance calculation after STED imaging. (d) Schematic of the workflow for distance calculation after STORM imaging. (e) Representative images of STORM imaging for FUS-GluN2B-Synapsin1 and FUS-PSD95-Bassoon. (f) Violin graph representing the distance distribution between FUS and synaptic markers. (g) Binning distribution showing the distance between FUS and the markers (in relative frequency) for PSD95, GluN2b, GluA1, Bassoon, Synapsin and BiP. 
1207 Supplementary Fig. 2 CLIP-seq on cortical synaptoneurosomes identified FUS1208 associated pre- and postsynaptic RNAs

1209 (a) Western blot of synaptic proteins (GluN2b, SNAP25, GluA1, NRXN1), nuclear protein 1210 (Histone H3) in total cortex and synaptoneurosomes (SNS). (b) Schematic of CLIP-seq 1211 workflow from total homogenate and SNS from mouse cortex. (c) Immunoblot showing 1212 efficient immunoprecipitation of FUS from total cortex and SNS. (d) Flow chart illustrating the 1213 reads analyzed to define FUS peaks in total and SNS. (e) MA-plot of CLIPper peaks 1214 predicted in the total cortex CLIP-seq sample. logCPM is the average log2CPM of each 1215 peak in the total cortex and SNS sample and logFC is the log2 fold-change between the 1216 number of reads in the total cortex and SNS sample. (f) Same MA-plot as (e) showing the 1217 selected, total cortex specific peaks ( $p$-value cutoff of $3 e-03$ ) in red. (g) Bar plot of different 1218 sets of SNS peaks and their location in genes. The $p$-value cutoff of each set is on the x-axis 1219 and no cutoff refers to the full list of all predicted SNS CLIPper peaks. The selected cutoff is 1220 in bold. (h) Bar plot of different sets of total cortex peaks and their location in genes. The p1221 value cutoff of each set is on the x-axis and no cutoff refers to the full list of all predicted 1222 SNS CLIPper peaks. The selected cutoff is in bold. (i) GO terms enriched among the 1223 synapse specific FUS RNA targets.

1224

1225

Supplementary Fig. 3 Increased synaptic FUS localization in Fus ${ }^{\Delta N L S /+}$ mice affect 1226 GABAergic synapses

1227 (a) Western blot of total FUS, full length FUS and actin in synaptoneurosomes isolated from 1228 1-month-old $\mathrm{Fus}^{+/+}$and Fus ${ }^{\Delta N L S /+}$ mice. (b) Quantification of total FUS and full length FUS 1229 levels in synaptoneurosomes from $\mathrm{Fus}^{+/+}$and $\mathrm{Fus} \mathrm{s}^{\Delta N L S /+}$ at 1 month of age. (c) Confocal 1230 images of the hippocampal CA1 area from 1-month-old mice showing higher level of FUS in 1231 the dendritic tree and synaptic compartment in Fus ${ }^{\Delta N L S /+}$ mouse-model. On the top, low 1232 magnification pictures show the dendritic area of pyramidal cells stained with FUS (green), 1233 MAP2 (dendritic marker, magenta), Synapsin 1 (Syn, Synaptic marker, Cyan) and DAPI 1234 (Blue). Red box indicates the area imaged in the high magnification images below. (d) 1235 Higher magnification equivalent to the area highlighted in red in (c). (e) Workflow for 1236 synaptic marker quantification. Molecular layer of CA1 hippocampal area was imaged by 1237 confocal microscopy. Z-stacks were imaged from top (higher Z step with specific signal) to 1238 bottom (last step with specific signal) with a Z-step of $0.5 \mu \mathrm{m}$. The 40 middle steps were 1239 used for quantification. Confocal images were then processed with Huygens professional 1240 software for deconvolution. Fiji was used for quantification. Images were first thresholded to 1241 only select the specific signal. Images were then binarized and quantification of size and 1242 density of synaptic markers was performed using the built-in "Analyze particles", with size 1243 exclusion threshold (as described in the Method section). Data were then compiled in open- 
1244 office and analyzed using Graphpad Prism software. (f) Heatmap summarizing the density of

1245 the different synaptic markers quantified in the CA1 hippocampal area from 1-month-old

$1246 F_{U S}{ }^{\Delta N L S /+}$ mice. Densities were normalized by the respective control. Mean value of each

1247 marker is indicated. Shade of color code for mean variation from 0 (white) to 2 (dark blue).

$1248{ }^{*} p<0.05$. (g) Heatmap summarizing the density of the different synaptic markers quantified in 1249 the CA1 hippocampal area from 6-month-old Fus ${ }^{\Delta N L S /+}$ mice. Densities were normalized by

1250 the respective control $\left(\mathrm{Fus}^{+/+}\right)$. Mean value of each marker is indicated. Shade of color code

1251 for mean variation from 0 (white) to 2 (dark blue). ${ }^{*} p<0.05$. (h) Heatmap summarizing the

1252 cluster area of the different synaptic markers quantified in the CA1 hippocampal area from 1-

1253 month-old $\mathrm{Fus}^{+/+}$and Fus ${ }^{\Delta N L S /+}$ mice. Mean value of each marker is indicated. Shade of color

1254 code for mean variation from 0.01 (white) to 1 (dark red). * $p<0.05$. (i) Heatmap summarizing

1255 the cluster area of the different synaptic markers quantified in the CA1 hippocampal area 1256 from 6-month-old Fus ${ }^{+/+}$and Fus ${ }^{\Delta N L S /+}$ mice. Mean value of each marker is indicated. Shade 1257 of color code for mean variation from 0.01 (white) to 1 (dark red). ${ }^{*} p<0.05{ }^{* *} p<0.01$.

Supplementary Fig. 4 Age-dependent alterations in the synaptic RNA profile of 1260 Fus ${ }^{\triangle N L S /+}$ mouse cortex.

1261 (a) Overlap between transcripts expressed in SNS RNA-seq and expressed genes in 1262 forebrain synaptic transcriptome reported previously ${ }^{99}$. Expressed genes are all genes with > 126310 reads in $2 / 3$ of the replicates (as defined previously ${ }^{99}$ ). (b) Plot of the first and second 1264 principal component of all RNA-seq samples and all expressed genes. The genotype is 1265 indicated by the symbol and the preparation and age by the color: 1-month-old mice in light and 6-month-old mice in dark colors. (c) Plot of the first and third principal component of all RNA-seq samples. (d) GO terms enriched among the significantly upregulated genes at 6 months of age in synaptoneurosomes of $\mathrm{Fus}^{\Delta N L S /+}$ compared to Fus ${ }^{+/+}$. (e) Gene ontology

1269 (GO) terms enriched among the significantly increased RNAs at 6 months of age in 1270 synaptoneurosomes of $\mathrm{Fus}^{\triangle N L S /+}$ compared to $\mathrm{Fus}^{+/+}$(f) Heatmap from the set of up- and 1271 downregulated genes between total cortex samples from $F u s^{\Delta N L S /+}$ and $F u s^{+/+}$at 6 months of 1272 age. Genes are on the rows and the different total cortex samples on the columns. The color 1273 scale indicates the log2FC between the CPM of each sample and mean CPM of the corresponding $\mathrm{Fus}^{+/+}$samples at each time point [sample logCPM - mean (logCPM of Fus ${ }^{+/+}$ samples)]. (g) Volcano plots showing the log2 fold change of each gene and the corresponding - $\log 10$ (FDR) of the differential gene expression analysis comparing total cortex from Fus ${ }^{\Delta N L S /+}$ to $\mathrm{Fus}^{+/+}$at 1 month (left panel) and 6 months (right panel) of age. The

1278 horizontal line marks the significance threshold of 0.05 . Significantly downregulated genes are highlighted in green, upregulated genes in purple. 
1281 Supplementary Fig. 5. FUS peak locations on presynaptic and transsynaptic FUS RNA

1282 targets altered in Fus ${ }^{\Delta N L S /+}$ mice.

1283 CLIP-traces showing FUS binding on (a) Syp (b) Robo2 (c) Sv2a (d) Syt1 (e) Chl1 (f) App

$1284 \quad$ (g) Aplp2

1285

1286

Supplementary Figure 6. FUS peak locations on postsynaptic FUS RNA targets

1287 altered in Fus ${ }^{\Delta N L S /+}$ mice.

1288 CLIP-traces showing FUS binding on (a) Gria2 (b) Gria3 (c) Atp1a1 (d) Atp1a3 (e) Atp1b1

1289 (f) Spock1 (g) Spock2 (h) Clstn1

1290

Supplementary Figure 7. FUS binding on Gabra1 RNA.

1292

CLIP-traces showing FUS binding to the long 3'UTR containing isoform of Gabra1

1293

1294

1295 
bioRxiv preprint doi: $h t t p \mathbf{b} /$ doi.org/10.1101/2020.06.10 36010; this version posted June $10 \mathbf{0 2 0}$. The copyrightholder for this preprint (which was not certified by peer review) is the author/funder, who has granted bioRxiv a license to display the preprintiqfaperpetuity. It is made SNS available under aCC-BY-NC-ND 4.0 International license.
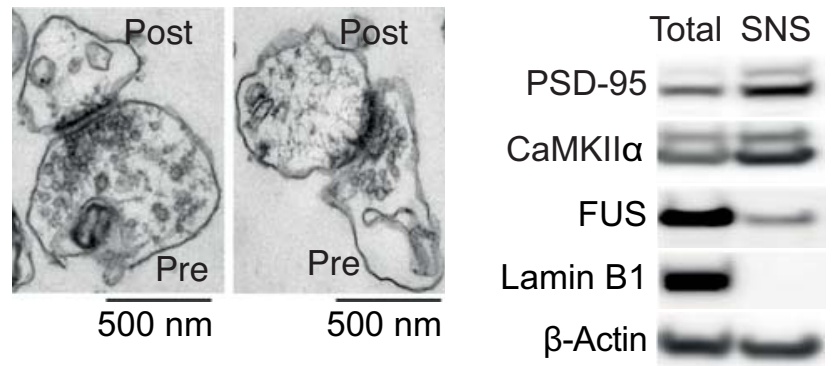

$\lg G$

IP FUS $\lg G$ FUS MNase $100 \mathrm{U} 0.02 \mathrm{U}-100 \mathrm{U} 0.02 \mathrm{U}$

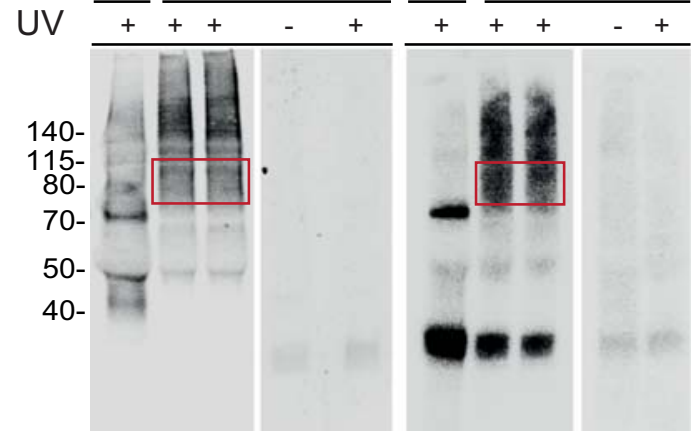

f

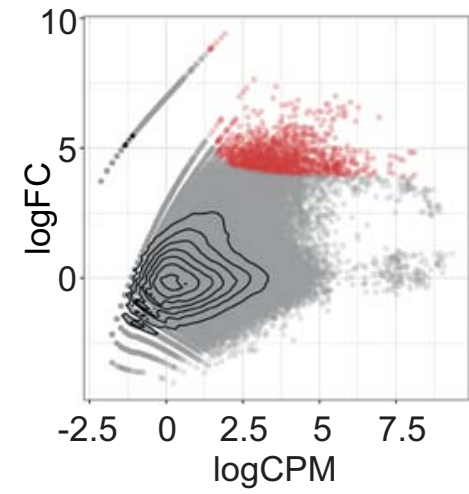

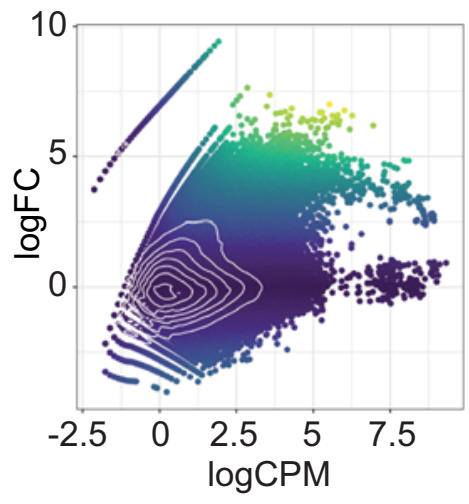

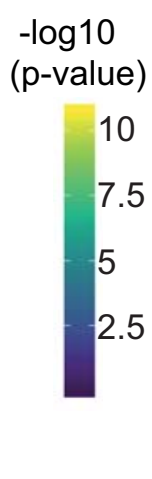

g

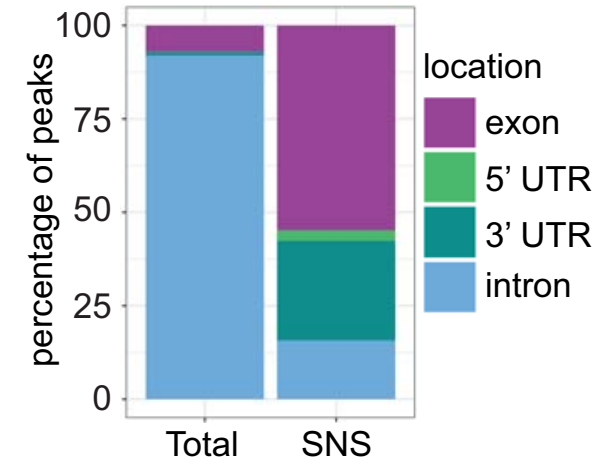

h

Glutamate ionotropic receptor NMDA type subunit 1 (Grin1)

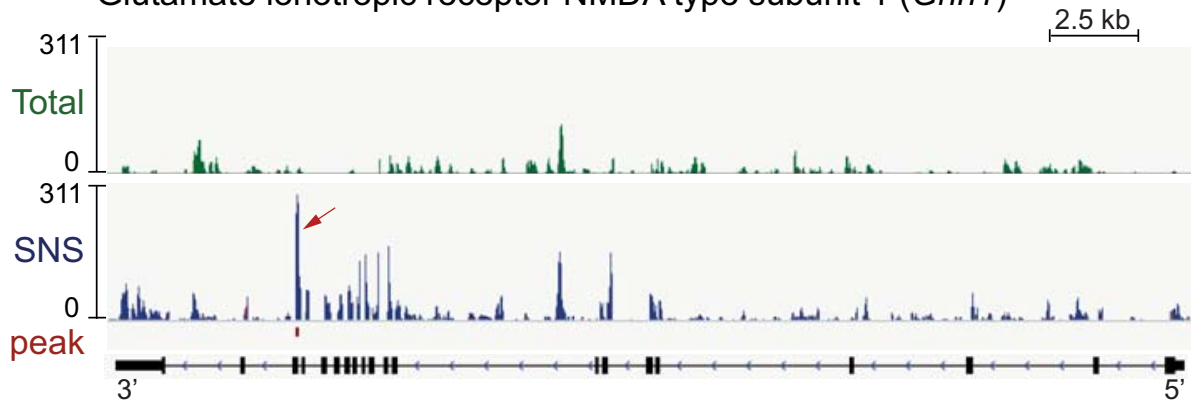

Gamma-aminobutyric acid type A receptor alpha1 subunit (Gabra1)

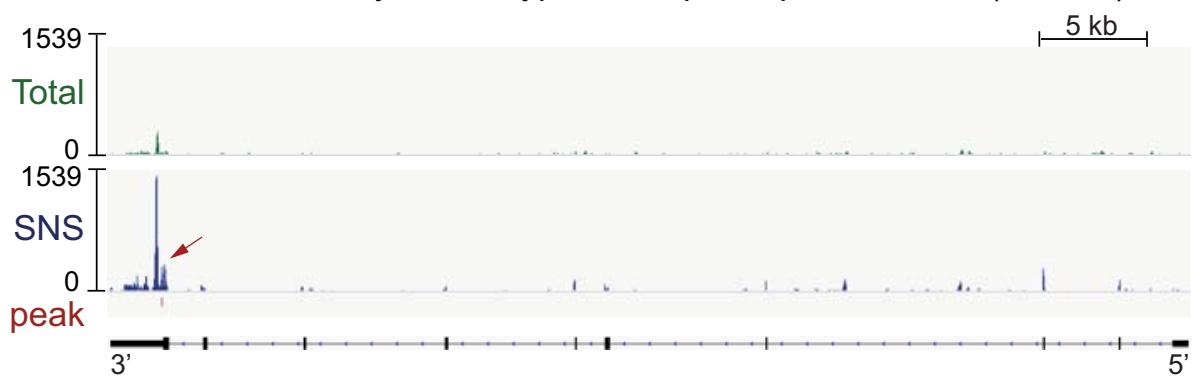

j

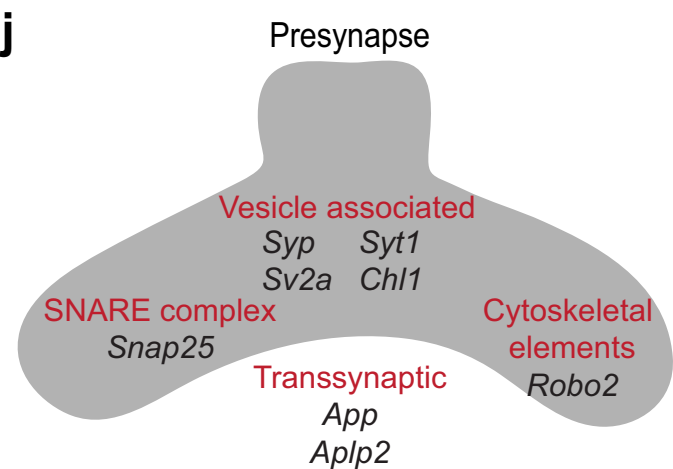

Glutamatergic GABAergic

Grin1

Gria2

Gria3

Gabra1

Gabrb3

Gabbr1

Transporters

Gabbr2

Atp1a1 Calcium binding Cytoskeletal

Atp1a3 Camk2a elements

Atp1b1 Camk2n1 Spock1

Slc6a11 Clstn1 Spock2

Slc6a1 Clstn2 Robo2

Postsynapse 
a Full length EloBixiv preprint doi: https://doi.org/10.1101/20R0.06.10 36010 ; this version posted June 10,2020 . The copyright holder foenis preprint QGSY (which was not

\section{FUS ${ }^{\triangle N L S}$}

QGSY rich G-rich RGG RRM RGG ZnF RGG

b

\begin{tabular}{|c|c|c|}
\hline & \multicolumn{2}{|c|}{ SNS - 6-month-old } \\
\hline & $\mathrm{Fus}^{+/+}$ & $F u s^{\Delta N L S /+}$ \\
\hline A Total FUS & - - - - in & 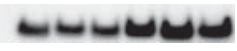 \\
\hline Full length FUS & ーーーールー & $-2-1-\infty$ \\
\hline
\end{tabular}

C

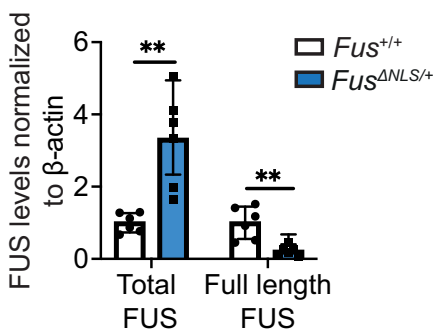

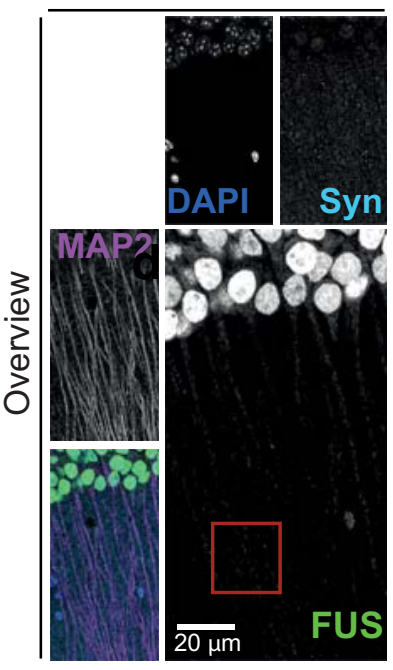

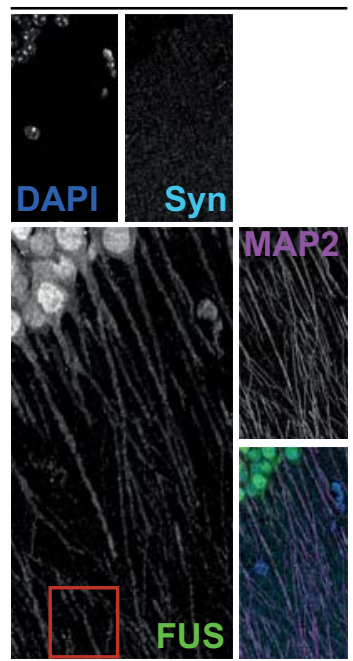

High magnification
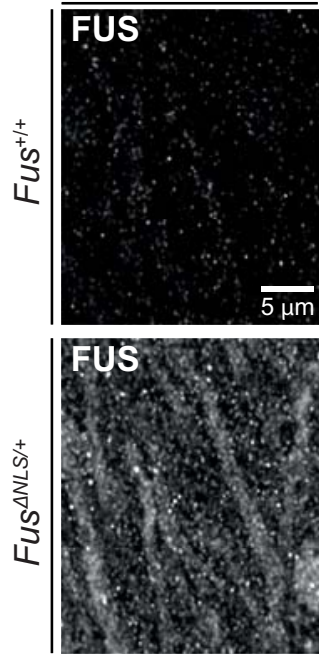

f
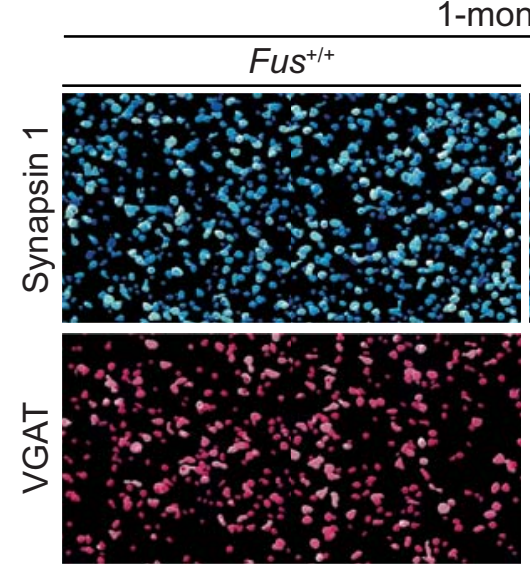

8
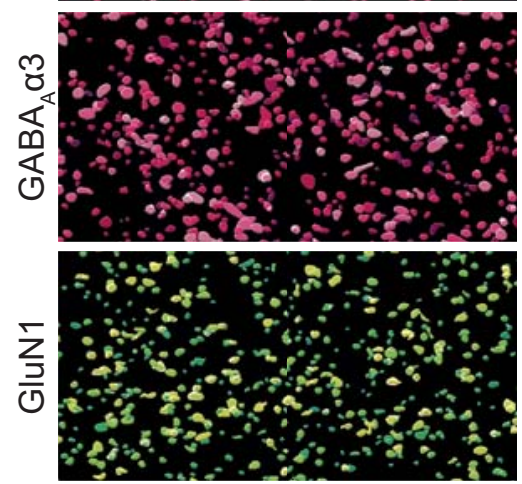

g

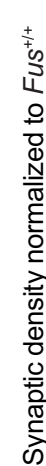$$
0
$$

0.0792

而
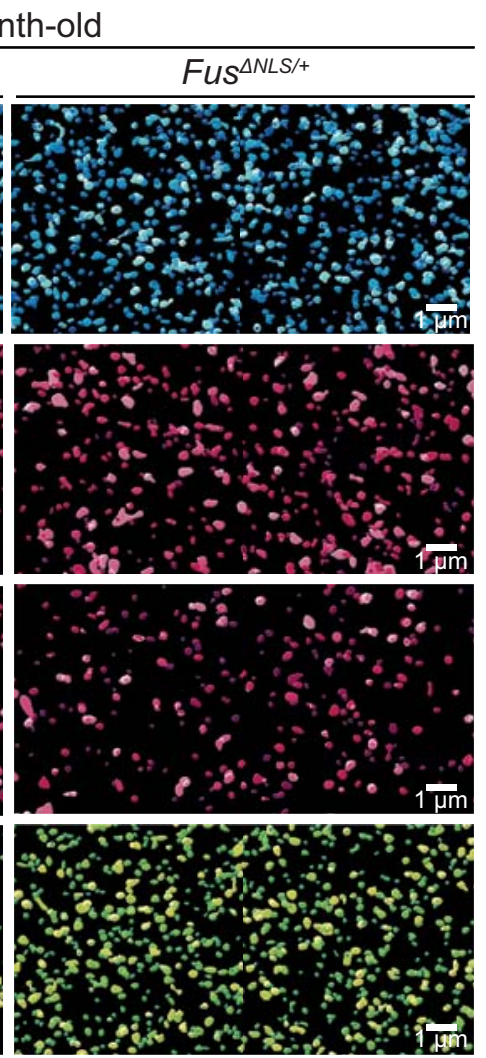

h

$\square \mathrm{Fus}^{+/+}$

$\square$ FUS $\triangle$ SLLS/+

$\stackrel{*}{-}$

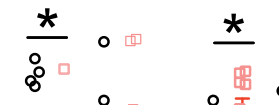

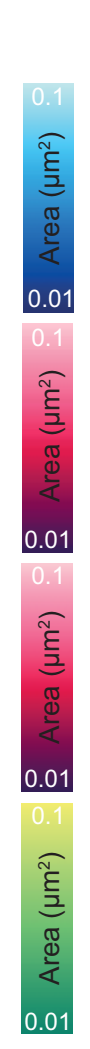
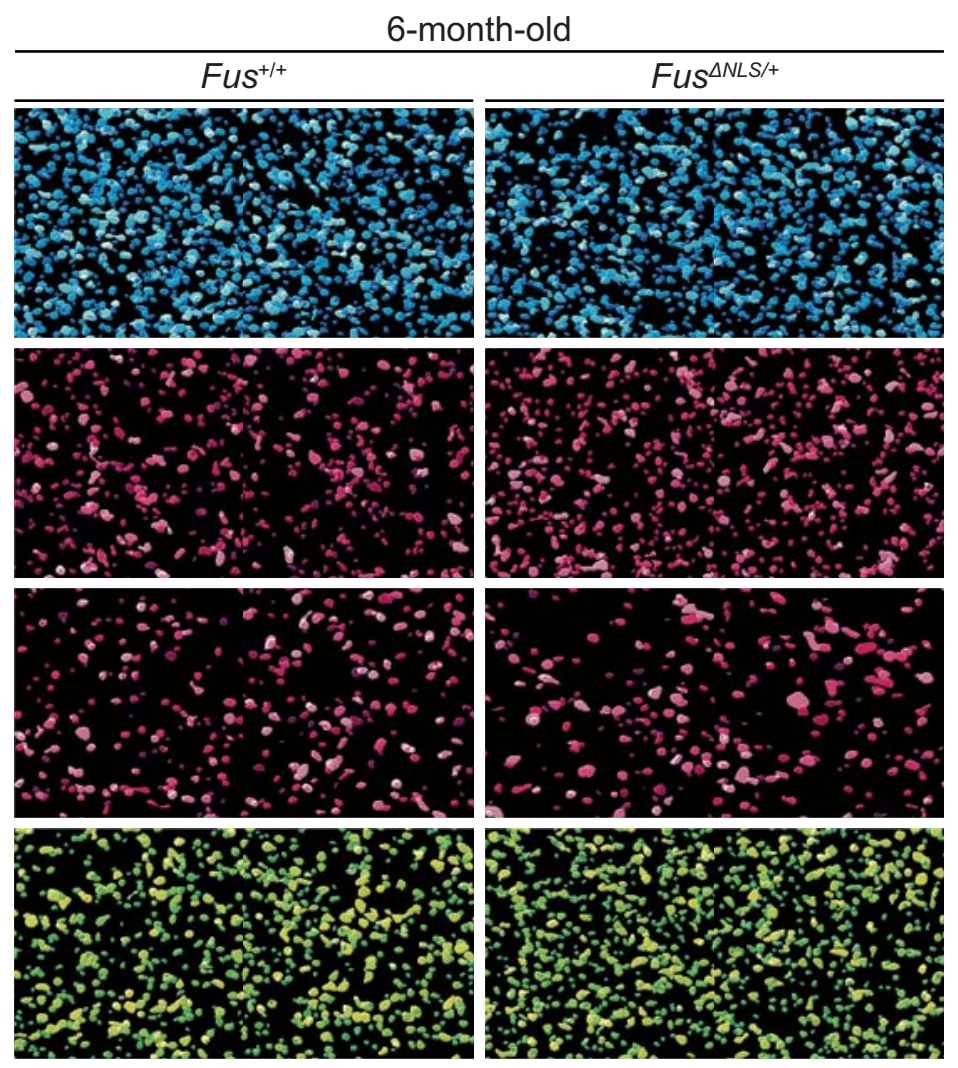

Fig 3. Increased synaptic FUS localization in Fus ${ }^{\Delta N L S /+}$ mice affect GABAergic synapses 


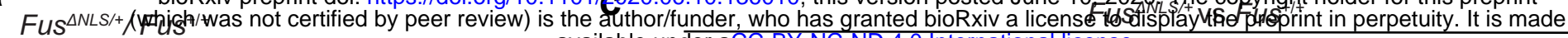

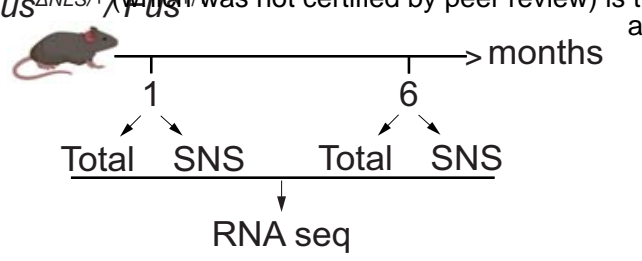

b

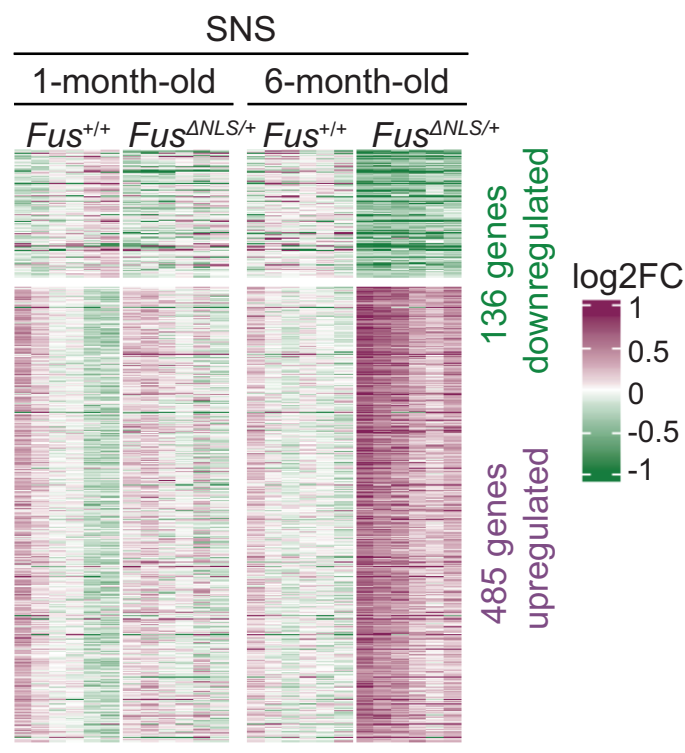

d

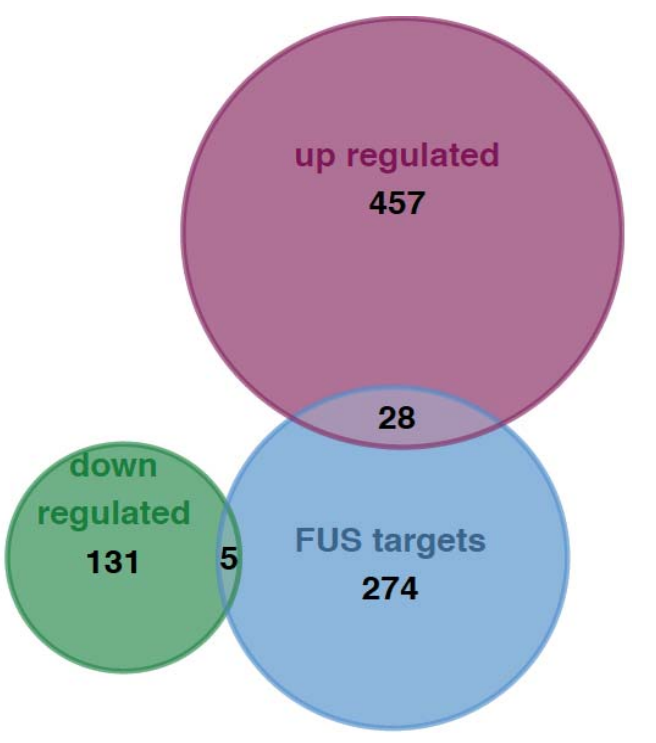

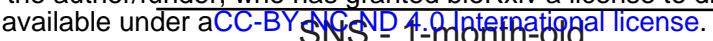
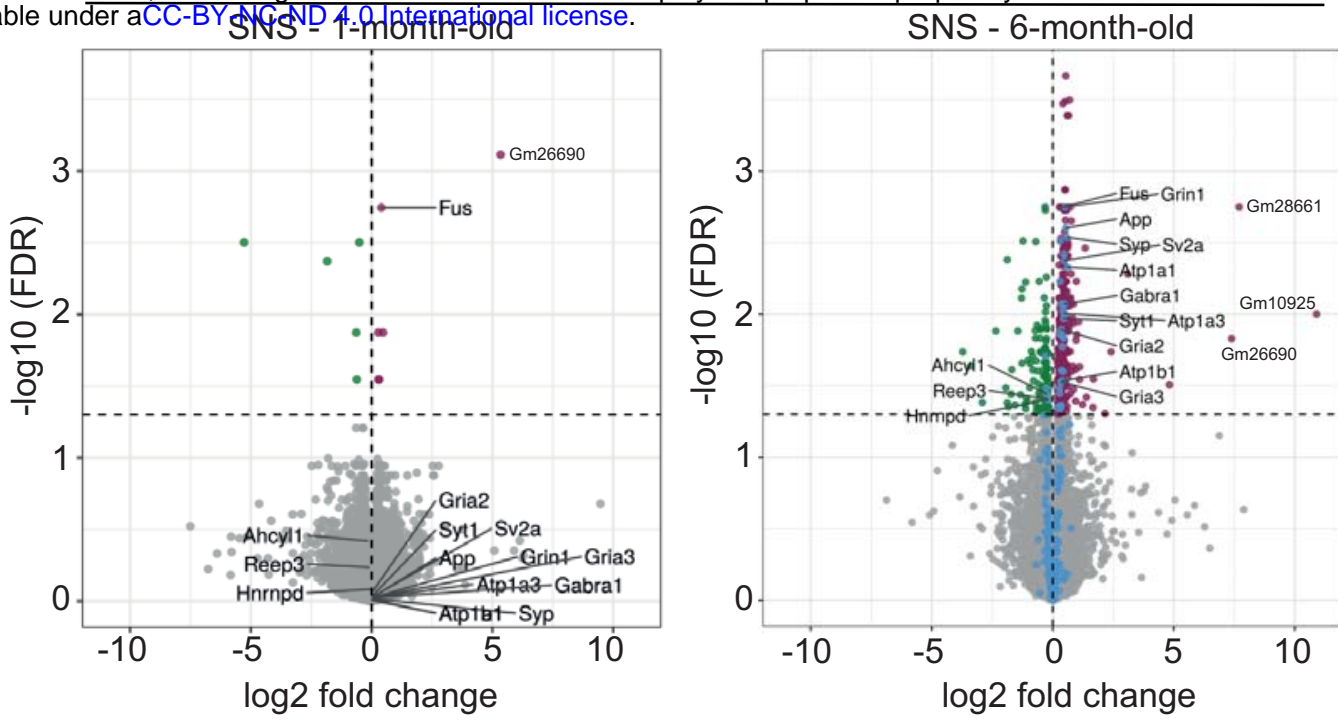

- FDR $>0.05$

- $\mathrm{FDR} \leq 0.05 \& \log \mathrm{FC}>0$

- FDR $\leq 0.05 \& \log F C<0$

\section{- FUS target}

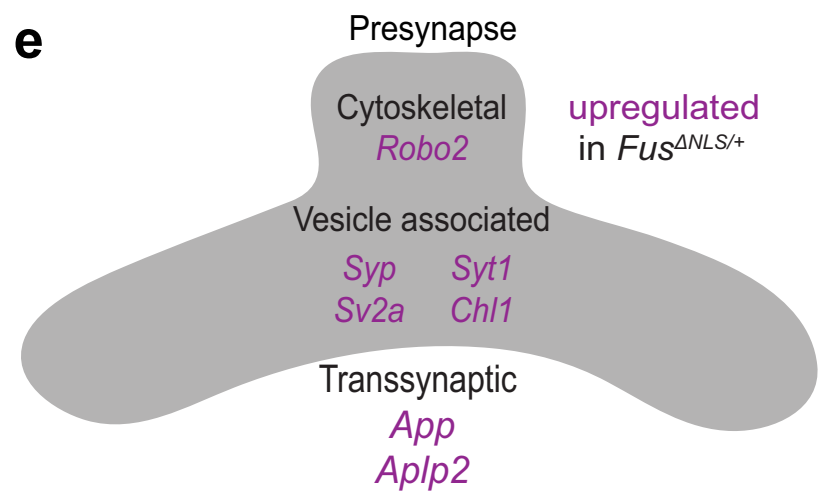

Glutamatergic

Grin1

Transporters $\quad$ Gria2 $\quad$ GABa3 $\quad$ Gabrgic

Atp1a1 Gabra1

Atp1a3 Cytoskelatal

Atp1b1 Spock1 Calcium binding

Spock2 Clstn1

Robo2

Postsynapse 
bioRxiv preprint doi: https://doi.org/10.1101/2020.06.10.136010; this version posted June 10, 2020. The copyright holder for this preprint (which was not certified by peer review) is the author/funder, who has granted bioRxiv a license to display the preprint in perpetuity. It is made

\begin{tabular}{|c|c|c|c|c|}
\hline \multirow{2}{*}{ Target sequences } & \multicolumn{3}{|c|}{ available under aCC-BY-NC-ND 4.0 International license. } & \multirow[b]{2}{*}{$\%$ of background } \\
\hline & Motif & $p$ value & $\%$ of targets & \\
\hline Total cortex, intron & & $1 e-17$ & 7.23 & 1.39 \\
\hline SNS, intron & & $1 e-13$ & 3.67 & 0.15 \\
\hline SNS, intron & & $1 e-12$ & 13.84 & 4.24 \\
\hline SNS, 3' UTR & & $1 \mathrm{e}-17$ & 7.23 & 1.39 \\
\hline SNS, exon & & $1 e-40$ & 10.83 & 1.46 \\
\hline SNS 5' UTR & & $1 e-15$ & 6.21 & 0.11 \\
\hline
\end{tabular}

Table 1: FUS binds GU-rich sequences at the synapse

Predicted sequence motifs (HOMER) in windows of size 41 centered on the position with maximum coverage in each peak. Each set of target sequences has a corresponding background set with 200,000 sequences without any CLIP-seq read coverage (they are not bound by FUS). Note: These are all motifs that were not marked as possible false positives by HOMER and that occur in more than $1 \%$ of the target sequences. 
bioRxiv preprint doi: https://doi.org/10.1101/2020.06.10.136010; this version posted June 10, 2020. The copyright holder for this preprint (which was not certified by peer review) is the author/funder, who has granted bioRxiv a license to display the preprint in perpetuity. It is made

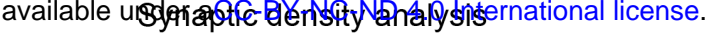

\begin{tabular}{|c|c|c|c|c|c|c|}
\hline unpaired t-test & \multicolumn{3}{|c|}{1 month } & \multicolumn{3}{|c|}{6 months } \\
\hline & $p$ value & $t, d f$ & sample size & $p$ value & $t, d f$ & sample size \\
\hline Synapsin1 & 0.4556 & $\mathrm{t}=0.7553, \mathrm{df}=32$ & $\begin{array}{r}+/+=14 \\
\Delta N L S /+=20\end{array}$ & 0.6812 & $t=0.4138, d f=41$ & $\begin{array}{r}+/+=19 \\
\Delta \mathrm{NLS} /+=24\end{array}$ \\
\hline SNAP25 & 0.5320 & $\mathrm{t}=0.6319, \mathrm{df}=32$ & $\begin{array}{r}+/+=14 \\
\Delta \mathrm{NLS} /+=20\end{array}$ & 0.085 & $t=1.765, d f=41$ & $\begin{array}{r}+/+=19 \\
\Delta \mathrm{NLS} /+=24\end{array}$ \\
\hline Bassoon & 0.5821 & $t=0.5567, d f=28$ & $\begin{array}{r}+/+=18 \\
\Delta N L S /+=12\end{array}$ & 0.4460 & $\mathrm{t}=0.7708, \mathrm{df}=35$ & $\begin{array}{r}+/+=18 \\
\Delta \mathrm{NLS} /+=19\end{array}$ \\
\hline VGAT & 0.6368 & $\mathrm{t}=0.4758, \mathrm{df}=40$ & $\begin{array}{r}+/+=19 \\
\Delta \mathrm{NLS} /+=23\end{array}$ & 0.0792 & $t=1.801, d f=40$ & $\begin{array}{r}+/+=18 \\
\Delta \mathrm{NLS} /+=24\end{array}$ \\
\hline GluN1 & 0.0219 & $t=2.409, d f=32$ & $\begin{array}{r}+/+=14 \\
\Delta N L S /+=20\end{array}$ & 0.3786 & $\mathrm{t}=0.8900, \mathrm{df}=41$ & $\begin{array}{r}+/+=19 \\
\Delta \mathrm{NLS} /+=24\end{array}$ \\
\hline GluA1 & 0.6009 & $\mathrm{t}=0.5292, \mathrm{df}=28$ & $\begin{array}{r}+/+=18 \\
\Delta \mathrm{NLS} /+=12\end{array}$ & 0.4885 & $t=0.7000, d f=35$ & $\begin{array}{r}+/+=18 \\
\Delta \mathrm{NLS} /+=19\end{array}$ \\
\hline pCaMKII & 0.9055 & $t=0.1195, d f=40$ & $\begin{array}{r}+/+=19 \\
\Delta \mathrm{NLS} /+=23\end{array}$ & 0.2160 & $t=1.257, d f=40$ & $\begin{array}{r}+/+=18 \\
\Delta \mathrm{NLS} /+=24\end{array}$ \\
\hline Gephyrin & 0.9878 & $\mathrm{t}=0.1531, \mathrm{df}=88$ & $\begin{array}{r}+/+=43 \\
\Delta N L S /+=47\end{array}$ & 0.5778 & $t=0.5591, d f=74$ & $\begin{array}{r}+/+=34 \\
\Delta \mathrm{NLS} /+=42\end{array}$ \\
\hline GABAAR $\alpha 1$ & 0.1368 & $t=1.514, d f=46$ & $\begin{array}{r}+/+=24 \\
\Delta \mathrm{NLS} /+=24\end{array}$ & 0.9611 & $\mathrm{t}=0.04906, \mathrm{df}=44$ & $\begin{array}{r}+/+=20 \\
\Delta \mathrm{NLS} /+=26\end{array}$ \\
\hline GABAAR $\alpha 3$ & 0.0156 & $t=2.512, d f=46$ & $\begin{array}{r}+/+=24 \\
\Delta \mathrm{NLS} /+=24\end{array}$ & 0.9744 & $t=0.03234, d f=40$ & $\begin{array}{r}+/+=20 \\
\Delta \mathrm{NLS} /+=22\end{array}$ \\
\hline
\end{tabular}

Table 2. Statistical analysis of synaptic density

The table reports statistical analysis of density of the synaptic markers analyzed from a minimum of 2 images from at least 4 animals per genotype $\left(F u s^{+/+}\right.$and $\left.F u s^{\Delta N L S /+}\right)$ at 1 and 6 months of age. Unpaired $\mathrm{t}$-test statistics, $\mathrm{p}$-values, specific $\mathrm{t}$-distribution ( $\mathrm{t}$ ), degrees of freedom (DF) and sample size are listed. 
bioRxiv preprint doi: https://doi.org/10.1101/2020.06.10.136010; this version posted June 10, 2020. The copyright holder for this preprint (which was not certified by peer review) is the author/funder, who has granted bioRxiv a license to display the preprint in perpetuity. It is made

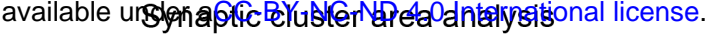

\begin{tabular}{|c|c|c|c|c|c|c|}
\hline unpaired t-test & \multicolumn{3}{|c|}{1 month } & \multicolumn{3}{|c|}{6 months } \\
\hline & $p$ value & $t, d f$ & sample size & $p$ value & $t, d f$ & sample size \\
\hline Synapsin1 & 0.8249 & $t=0.2214, d f=363$ & $\begin{array}{r}+/+=14 \\
\Delta \mathrm{NLS} /+=20\end{array}$ & 0.643 & $t=0.4639, d f=393$ & $\begin{array}{r}+/+=19 \\
\Delta \mathrm{NLS} /+=24\end{array}$ \\
\hline SNAP25 & 0.3834 & $t=0.8727, d f=363$ & $\begin{array}{r}+/+=14 \\
\Delta \mathrm{NLS} /+=20\end{array}$ & 0.5015 & $t=0.6727, d f=393$ & $\begin{array}{r}+/+=19 \\
\Delta \mathrm{NLS} /+=24\end{array}$ \\
\hline Bassoon & 0.6022 & $\mathrm{t}=0.5217, \mathrm{df}=363$ & $\begin{array}{r}+/+=18 \\
\Delta \mathrm{NLS} /+=12\end{array}$ & 0.7529 & $t=0.315 d f=393$ & $\begin{array}{r}+/+=18 \\
\Delta \mathrm{NLS} /+=19\end{array}$ \\
\hline VGAT & 0.2819 & $t=1.078, d f=363$ & $\begin{array}{r}+/+=19 \\
\Delta \mathrm{NLS} /+=23\end{array}$ & 0.0028 & $t=3.005, d f=393$ & $\begin{array}{r}+/+=18 \\
\Delta \mathrm{NLS} /+=24\end{array}$ \\
\hline GluN1 & 0.5437 & $t=6078, d f=363$ & $\begin{array}{r}+/+=14 \\
\Delta \mathrm{NLS} /+=20\end{array}$ & 0.5694 & $t=0.5694, d f=393$ & $\begin{array}{r}+/+=19 \\
\Delta \mathrm{NLS} /+=24\end{array}$ \\
\hline GluA1 & 0.4303 & $t=0.7896, d f=363$ & $\begin{array}{r}+/+=18 \\
\Delta \mathrm{NLS} /+=12\end{array}$ & 0.4517 & $t=0.7533, d f=393$ & $\begin{array}{r}+/+=18 \\
\Delta \mathrm{NLS} /+=19\end{array}$ \\
\hline pCaMKII & 0.242 & $t=1.172, d f=363$ & $\begin{array}{r}+/+=19 \\
\Delta \mathrm{NLS} /+=23\end{array}$ & 0.4150 & $t=0.8159, d f=393$ & $\begin{array}{r}+/+=18 \\
\Delta \mathrm{NLS} /+=24\end{array}$ \\
\hline Gephyrin & 0.7467 & $t=0.3233, d f=363$ & $\begin{array}{r}+/+=43 \\
\Delta \mathrm{NLS} /+=47\end{array}$ & 0.2614 & $t=1.125, d f=393$ & $\begin{array}{r}+/+=34 \\
\Delta \mathrm{NLS} /+=42\end{array}$ \\
\hline GABAAR $\alpha 1$ & 0.374 & $\mathrm{t}=0.8902, \mathrm{df}=363$ & $\begin{array}{r}+/+=24 \\
\Delta \mathrm{NLS} /+=24\end{array}$ & 0.3204 & $t=0.9950 \mathrm{df}=393$ & $\begin{array}{r}+/+=20 \\
\Delta \mathrm{NLS} /+=26\end{array}$ \\
\hline GABAAR $\alpha 3$ & 0.0053 & $t=2.807, d f=363$ & $\begin{array}{r}+/+=24 \\
\Delta \mathrm{NLS} /+=24\end{array}$ & 0.0166 & $t=2.407, d f=393$ & $\begin{array}{r}+/+=20 \\
\Delta \mathrm{NLS} /+=22\end{array}$ \\
\hline
\end{tabular}

Table 3. Statistical analysis of synaptic cluster area

The table reports statistical analysis of area of the synaptic markers analyzed from a minimum of 2 images from at least 4 animals per genotype $\left(F u s^{+/+}\right.$and $\left.F u s^{\Delta N L S /+}\right)$ at 1 and 6 months of age. Unpaired t-test statistics, p-values, specific t-distribution ( $t$ ), degrees of freedom (DF) and sample size are listed. 